\title{
Nickel Catalysis in a High Speed Ball Mill: A Recyclable Mechanochemical Method for Producing Substituted Cyclooctatetraene Compounds
}

Rebecca A. Haley, Aaron R. Zellner, Jeanette A. Krause, Hairong Guan*, and James Mack*

\author{
Department of Chemistry, University of Cincinnati, Cincinnati, Ohio, 45221-0172 \\ *To whom correspondence should be addressed \\ Emails: hairong.guan@uc.edu; james.mack@uc.edu
}

\section{SUPPLEMENTARY INFORMATION (PAGES S1-S34)}

CONTENTS (contains 18 figures and 5 tables)

Characterization data for cyclooctatetraene compounds...........................S2 7

${ }^{1} \mathrm{H}$ and ${ }^{13} \mathrm{C}\left\{{ }^{1} \mathrm{H}\right\}$ NMR spectra for cyclooctatetraene compounds......................S8-S25

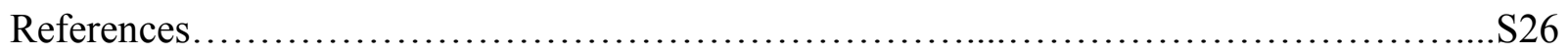

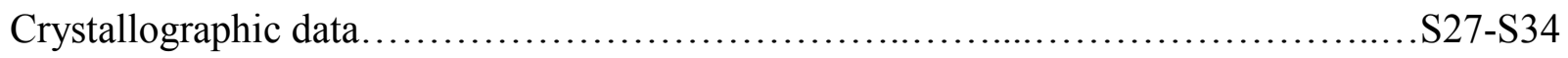


Cyclotrimerization products $\mathbf{2}(\mathbf{a}-\mathbf{d}, \mathbf{h}-\mathbf{i}),{ }^{1} \mathbf{2} \mathbf{f},{ }^{2} \mathbf{2}(\mathbf{e}, \mathbf{g}),{ }^{3} \mathbf{3}(\mathbf{a}-\mathbf{e}, \mathbf{g - i}),{ }^{4}$ and $\mathbf{3 f ^ { 5 }}$ have been reported in the literature and are consistent with the reported data.

\section{Characterization data for COT compounds:}

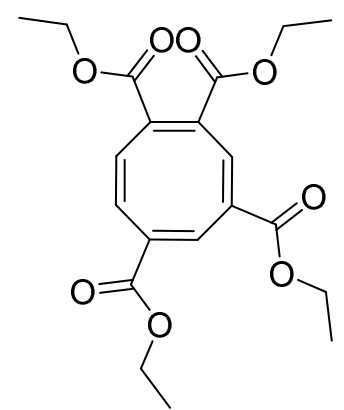

$4 \mathbf{a}$<smiles>CCOC(=O)C1=CC(C(=O)OCC)=CC(C(=O)OCC)=C(C(=O)OCC)C=C1</smiles>

5a<smiles>CCOC(=O)C1=CC(C(=O)OCC)=CC(C(=O)OCC)=CC(C(=O)OCC)=C1</smiles>

$7 \mathbf{a}$

\section{Ethyl esters (4a, 5a, 7a)}

Purification from the benzene derivatives by silica flash column chromatography ( $80: 20$ petroleum ether/ethyl acetate) yielded the mixture of ethyl ester substituted COT isomers in $61 \%$ combined yield. ${ }^{1} \mathrm{H} \mathrm{NMR}\left(\mathrm{CDCl}_{3}, 400 \mathrm{MHz}, \delta\right): 7.38(\mathrm{~s}, 1 \mathrm{H}$ of $4 \mathbf{a}), 7.36(\mathrm{~d}, J=0.8 \mathrm{~Hz}, 2 \mathrm{H}$ of 5a), $7.32(\mathrm{~s}, 1 \mathrm{H}$ of $\mathbf{4 a}), 7.30(\mathrm{~s}, 4 \mathrm{H}$ of $\mathbf{7 a}), 7.30(\mathrm{~d}, J=4.0 \mathrm{~Hz}, 1 \mathrm{H}$ of $\mathbf{4 a}), 7.21$ (dd, $J=4.0,1.3$ $\mathrm{Hz}, 1 \mathrm{H}$ of $\mathbf{4 a}), 7.16(\mathrm{~d}, J=0.8 \mathrm{~Hz}, 1 \mathrm{H}$ of $\mathbf{5 a}), 4.30-4.11\left(\mathrm{~m}, \mathrm{CH}_{2}\right.$ of $\mathbf{4 a}, \mathbf{5 a}$ and 7a), 1.33-1.22 (m, $\mathrm{CH}_{3}$ of $\mathbf{4 a}, \mathbf{5 a}$ and $\left.7 \mathbf{a}\right) ;{ }^{13} \mathrm{C}\left\{{ }^{1} \mathrm{H}\right\} \operatorname{NMR}\left(\mathrm{CDCl}_{3}, 101 \mathrm{MHz}, \delta\right)$ of $\mathbf{4 a}, \mathbf{5 a}$ and 7a: 164.77, 164.76, $164.48,164.42$, 164.33, 141.15, 139.95, 139.87, 139.63, 139.50, 139.20, 134.68, 134.43, 133.71, $133.56,133.26,132.84,61.90,61.85,61.71,61.53,61.46,61.38,14.33,14.24,14.22$; HRMS$\operatorname{ESI}(\mathrm{m} / \mathrm{z})[\mathrm{M}+\mathrm{Na}]^{+}$calcd for $\mathrm{C}_{20} \mathrm{H}_{24} \mathrm{O}_{8} \mathrm{Na} 415.1363$, found 415.1364 .<smiles>COC(=O)C1=CC(C(=O)OC)=CC(C(=O)OC)=C(C(=O)OC)C=C1</smiles>

4b<smiles>COC(=O)C1=CC(C(=O)OC)=CC(C(=O)OC)=C(C(=O)OC)C=C1</smiles>

$\mathbf{5 b}$<smiles>COC(=O)C1=C(C(=O)OC)C=CC(C(=O)OC)=C(C(=O)OC)C=C1</smiles>

6b<smiles>COC(=O)C1=CC(C(=O)OC)=CC(C(=O)OC)=CC(C(=O)OC)=C1</smiles>

$7 \mathbf{b}$

Methyl esters (4b-7b)

Purification from the benzene derivatives by silica flash column chromatography $(80: 20$ petroleum ether/ethyl acetate) yielded the mixture of methyl ester substituted COT isomers in $66 \%$ combined yield. ${ }^{1} \mathrm{H} \mathrm{NMR}\left(\mathrm{CDCl}_{3}, 400 \mathrm{MHz}, \delta\right): 7.40$ (s, $4 \mathrm{H}$ of $\left.\mathbf{6 b}\right), 7.39$ (s, 2H of 5b), 7.38 (s, $4 \mathrm{H}$ of $7 \mathbf{b}), 7.35$ (s, $1 \mathrm{H}$ of $\mathbf{4 b}), 7.33$ (d, $J=4.0 \mathrm{~Hz}, 1 \mathrm{H}$ of $\mathbf{4 b}), 7.27$ (s, 1H of $\mathbf{4 b}), 7.26$ $(\mathrm{dd}, J=4.0,1.2 \mathrm{~Hz}, 1 \mathrm{H}$ of $\mathbf{4 b}), 7.20(\mathrm{~s}, 2 \mathrm{H}$ of $\mathbf{5 b}), 3.82-3.75\left(4 \mathrm{~s}, \mathrm{CH}_{3}\right.$ of $\left.\mathbf{4 b}-\mathbf{7 b}\right) ;{ }^{13} \mathrm{C}\left\{{ }^{1} \mathrm{H}\right\} \mathrm{NMR}$ 
$\left(\mathrm{CDCl}_{3}, 101 \mathrm{MHz}, \delta\right)$ of $\mathbf{4 b - 7 b}: 165.14,165.12,165.11,164.80,164.75,164.63,141.29,140.26$, $140.10,139.92,139.76,139.54,134.29,133.95,133.38,133.29,132.89,132.64,52.83,52.77$, 52.72, 52.67, 52.57, 52.55; HRMS-ESI $(\mathrm{m} / \mathrm{z})[\mathrm{M}+\mathrm{Na}]^{+}$calcd for $\mathrm{C}_{16} \mathrm{H}_{16} \mathrm{O}_{8} \mathrm{Na} 359.0737$, found 359.0738 .

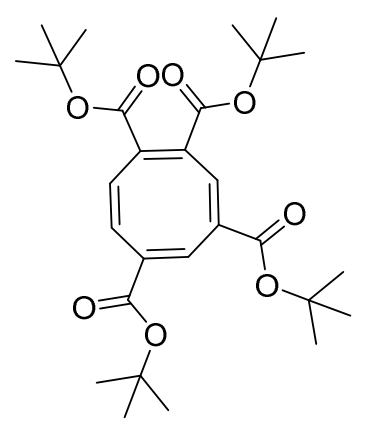

$4 \mathrm{c}$

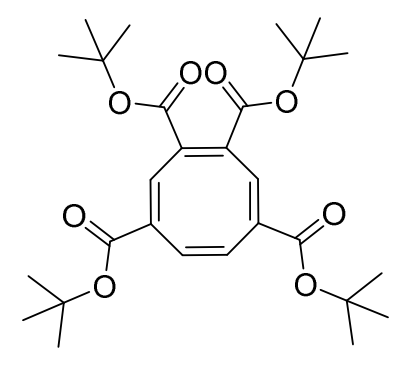

$5 c$

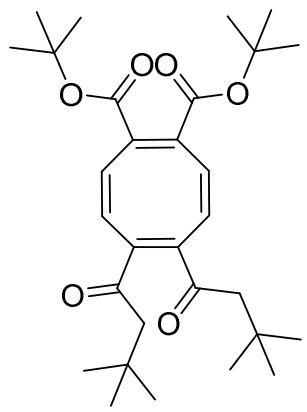

6c

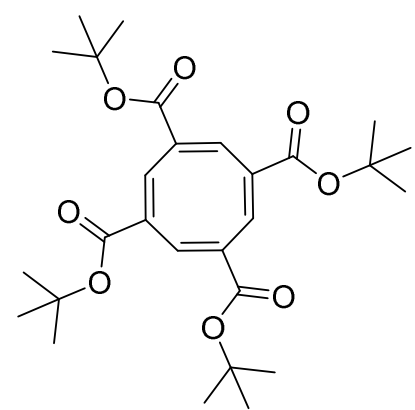

$7 \mathrm{c}$

\section{$t$-Butyl esters (4c-7c)}

Purification from the benzene derivatives by silica flash column chromatography (95: 5 petroleum ether/ethyl acetate) yielded the mixture of $t$-butyl ester substituted COT isomers in $75 \%$ combined yield. ${ }^{1} \mathrm{H}$ NMR $\left(\mathrm{CDCl}_{3}, 400 \mathrm{MHz}, \delta\right.$ ): 7.20 (s, $1 \mathrm{H}$ of $\left.4 \mathrm{c}\right), 7.18$ (s, $4 \mathrm{H}$ of $\mathbf{6 c}$ ), 7.15 $(\mathrm{s}, 1 \mathrm{H}$ of $4 \mathrm{c}), 7.12(\mathrm{~s}, 1 \mathrm{H}$ of $4 \mathrm{c}), 7.11(\mathrm{~s}, 2 \mathrm{H}$ of $\mathbf{5 c}), 7.10(\mathrm{~s}, 4 \mathrm{H}$ of $7 \mathrm{c}), 7.0(\mathrm{~s}, 2 \mathrm{H}$ of $\mathbf{5 c}), 6.99(\mathrm{~d}$, $J=1.2 \mathrm{~Hz}, 1 \mathrm{H}$ of $4 \mathrm{c}) 1.43-1.39\left(5 \mathrm{~s}, \mathrm{CH}_{3}\right.$ of $\left.4 \mathrm{c}-7 \mathrm{c}\right) ;{ }^{13} \mathrm{C}\left\{{ }^{1} \mathrm{H}\right\} \mathrm{NMR}\left(\mathrm{CDCl}_{3}, 101 \mathrm{MHz}, \delta\right)$ of $4 \mathrm{c}-$ 7c: $164.16,164.14,163.10,163.74,163.69,140.81,140.49,139.07,139.03,138.92,138.65$, $138.35,138.00,136.16,135.99,134.80,134.69,134.44,134.04,133.83,82.34,82.13,82.07$, $81.82,81.78,81.44,81.13,28.15,28.12,28.07$; HRMS-ESI $(\mathrm{m} / \mathrm{z})[\mathrm{M}+\mathrm{Na}]^{+}$calcd for $\mathrm{C}_{28} \mathrm{H}_{40} \mathrm{O}_{8} \mathrm{Na}$ 527.2615, found 527.2615.

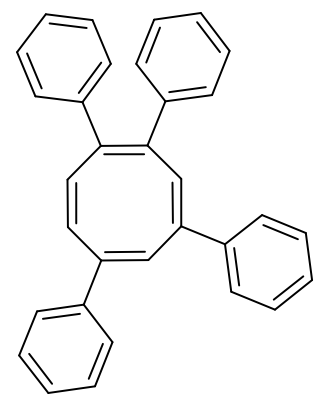

4d

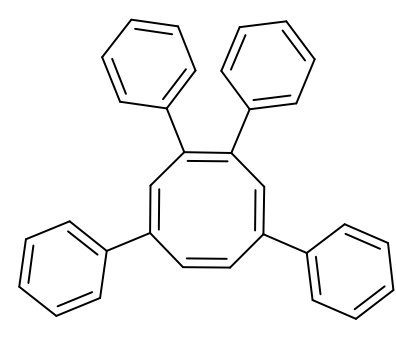

5d

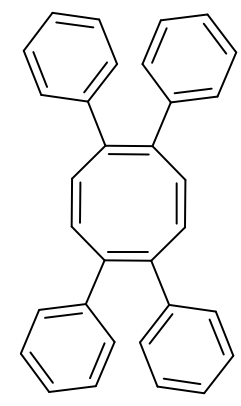

6d

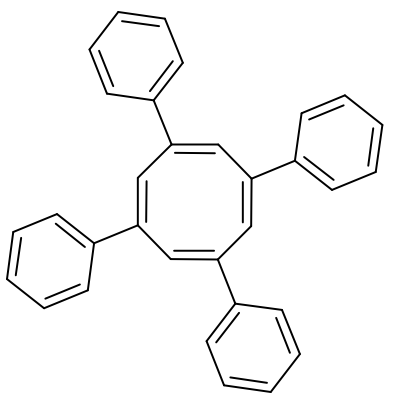

$7 d$

\section{Phenyl substituted COTs (4d, 5d, 6d (or 7d))}

Purification from the benzene derivatives by silica flash column chromatography $(100 \%$ petroleum ether) yielded the mixture of phenyl substituted COT isomers in 70\% combined yield. ${ }^{1} \mathrm{H}$ NMR $\left(\mathrm{CDCl}_{3}, 400 \mathrm{MHz}, \delta\right.$ ): 7.59-7.03 (m, $\mathrm{ArH}$ of 4d, 5d, 6d (or 7d)), 6.82 (s, 2H of 5d), $6.80(\mathrm{~s}, 1 \mathrm{H}$ of $\mathbf{4 d}), 6.74(\mathrm{~d}, J=4.0 \mathrm{~Hz}, 1 \mathrm{H}$ of $\mathbf{4 d}), 6.72(\mathrm{~s}, 4 \mathrm{H}$ of $\mathbf{6 d}$ or $7 \mathbf{d}), 6.63(\mathrm{~s}, 1 \mathrm{H}$ of $\mathbf{4 d})$, 6.54 (s, $1 \mathrm{H}$ of $\mathbf{4 d}$ ), 6.53 (s, $2 \mathrm{H}$ of $\mathbf{5 d}$ ); ${ }^{13} \mathrm{C}\left\{{ }^{1} \mathrm{H}\right\} \mathrm{NMR}\left(\mathrm{CDCl}_{3}, 101 \mathrm{MHz}, \delta\right)$ of $\mathbf{4 d}, \mathbf{5 d}, \mathbf{6 d}$ (or 7d): 144.60, 143.82, 143.44, 142.91, 142.80, 142.22, 141.57, 140.27, 139.95, 139.81, 139.78, 139.68, 
139.67, 130.34, 130.13, 130.11, 129.43, 128.72, 128.68, 128.55, 128.52, 128.46, 128.37, 128.13, $128.00,127.95,127.85,127.81,127.55,127.50,127.34,127.14,127.05,126.79,126.74,126.71$, 126.38, 126.27; HRMS-ESI (m/z) $\left[\mathrm{M}+\mathrm{Ag}^{107}\right]^{+}$calcd for $\mathrm{C}_{32} \mathrm{H}_{24} \mathrm{Ag} 515.0924$, found 515.0924.

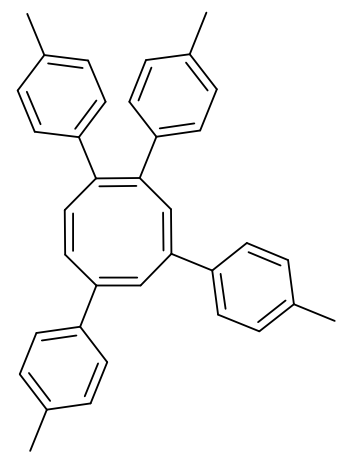

$4 e$

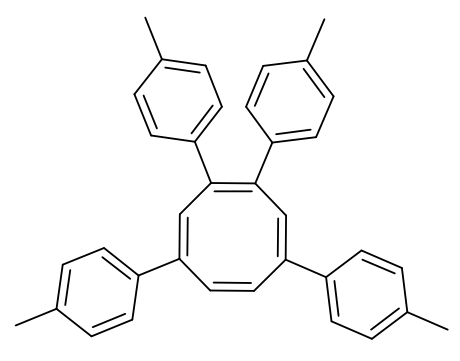

$5 \mathbf{e}$

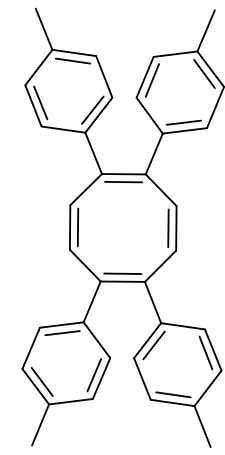

$6 e$

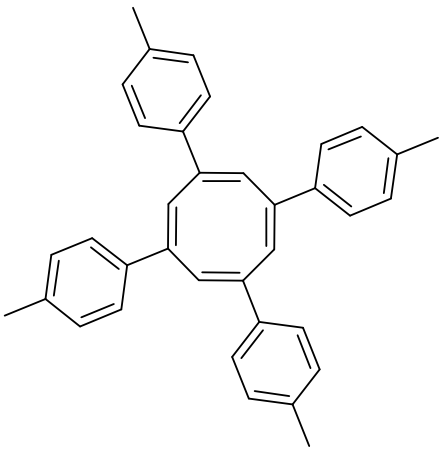

$7 e$

\section{$p$-Tolyl substituted COTs (4e, 5e, 6e (or 7e))}

Purification from the benzene derivatives by silica flash column chromatography $(100 \%$ petroleum ether) yielded the mixture of $p$-tolyl substituted COT isomers in 35\% combined yield. ${ }^{1} \mathrm{H} \mathrm{NMR}\left(\mathrm{CDCl}_{3}, 400 \mathrm{MHz}, \delta\right.$ ): 7.55-6.98 (m, $\mathrm{ArH}$ of 4e, 5e, 6e (or 7e)), 6.83 (s, 2H of 5e), 6.81 $(\mathrm{s}, 1 \mathrm{H}$ of $4 \mathrm{e}), 6.75(\mathrm{~d}, J=4.0 \mathrm{~Hz}, 1 \mathrm{H}$ of $4 \mathrm{e}), 6.73(\mathrm{~s}, 4 \mathrm{H}$ of $6 \mathbf{e}$ or $7 \mathrm{e}), 6.63(\mathrm{~s}, 1 \mathrm{H}$ of $4 \mathrm{e}), 6.56(\mathrm{~s}$, $2 \mathrm{H}$ of $5 \mathbf{e}), 6.55(\mathrm{~d}, J=4.0 \mathrm{~Hz}, 1 \mathrm{H}$ of $4 \mathbf{e}), 2.38-2.22\left(6 \mathrm{~s}, \mathrm{CH}_{3}\right.$ of $4 \mathbf{e}, 5 \mathbf{e}, 6 \mathbf{e}($ or $\left.7 \mathbf{e})\right) ;{ }^{13} \mathrm{C}\left\{{ }^{1} \mathrm{H}\right\}$ NMR $\left(\mathrm{CDCl}_{3}, 101 \mathrm{MHz}, \delta\right)$ of $4 \mathbf{e}, \mathbf{5 e}, \mathbf{6 e}$ (or 7e):144.24, 143.15, 142.65, 142.56, 141.96, 141.38, 137.69, 137.66, 137.49, 137.45, 137.33, 137.15, 137.12, 137.02, 136.98, 136.87, 136.84, 129.38, 129.34, 129.31, 129.20, 129.19, 129.17, 129.14, 129.05, 127.27, 127.07, 126.94, 126.85, 126.76, 126.60, 126.58, 126.51, 126.25, 126.14, 115.78, 21.32, 21.27, 21.23, 21.21; HRMS-ESI (m/z) $[\mathrm{M}+\mathrm{Na}]^{+}$calcd for $\mathrm{C}_{36} \mathrm{H}_{32} \mathrm{Na}$ 487.2402, found 487.2409.

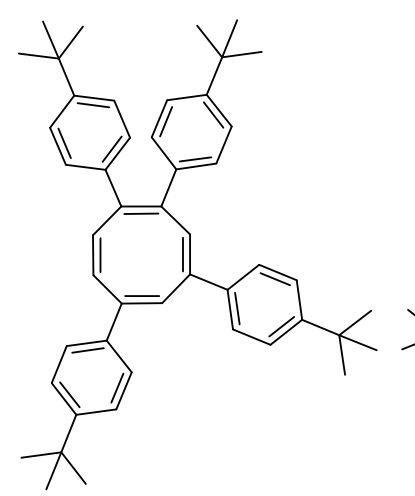

4f

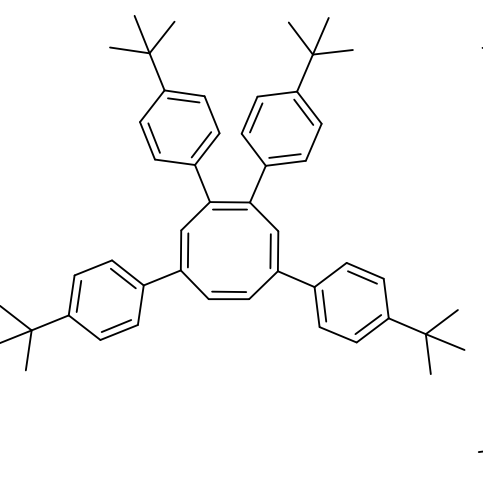

$5 f$

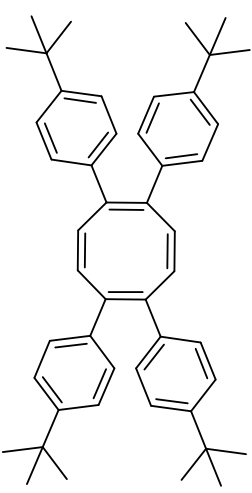

6f

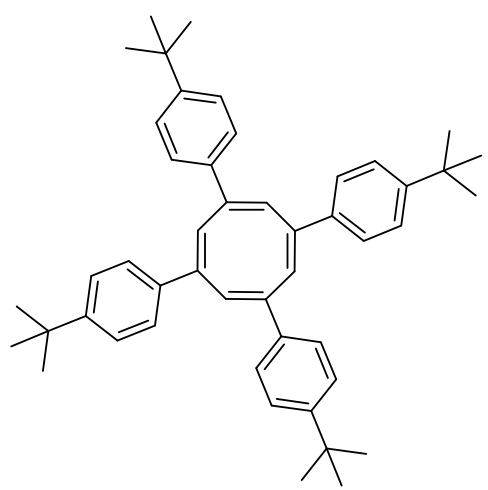

$7 \mathbf{f}$

\section{p-tert-Butylphenyl substituted COTs (4f, 5f, 6f (or 7f))}

Purification from the benzene derivatives by silica flash column chromatography $(100 \%$ petroleum ether) yielded the mixture of $p$-tert-butylphenyl substituted COT isomers in $14 \%$ combined yield. ${ }^{1} \mathrm{H}$ NMR $\left(\mathrm{CDCl}_{3}, 400 \mathrm{MHz}, \delta\right.$ ): 7.61-7.21 (m, $\mathrm{Ar} H$ of $\mathbf{4 f}, \mathbf{5 f}, \mathbf{6 f}$ (or 7f)), 6.86 (s, $2 \mathrm{H}$ of $\mathbf{5 f}$ ), 6.84 (s, $1 \mathrm{H}$ of $\mathbf{4 f}), 6.79$ (d, $J=4.0 \mathrm{~Hz}, 1 \mathrm{H}$ of $\mathbf{4 f}), 6.76$ (s, $4 \mathrm{H}$ of $\mathbf{6 f}$ or $\mathbf{7 f}$ ), 6.67 (s, $1 \mathrm{H}$ 
of 4e), $6.58(\mathrm{~d}, J=4.0 \mathrm{~Hz}, 1 \mathrm{H}$ of $\mathbf{2 f}), 6.57$ (s, 2H of $\mathbf{5 f}$ ), 1.34-1.24 (5s, $\mathrm{CH}_{3}$ of $\mathbf{4 f}, \mathbf{5 f}$, $6 \mathbf{f}$ ( or 7f)); ${ }^{13} \mathrm{C}\left\{{ }^{1} \mathrm{H}\right\} \mathrm{NMR}\left(\mathrm{CDCl}_{3}, 101 \mathrm{MHz}, \delta\right)$ of $\mathbf{4 f}, \mathbf{5 f}, \mathbf{6 f}$ (or 7f): 150.91, 150.69, 150.25, 150.21, 150.01, $144.17,142.99,142.44,142.34,141.85,141.28,137.64,137.26,137.05,137.00,136.92,136.91$, $127.21,127.13,126.97,126.56,126.39,126.25,126.15,126.10,125.97,125.58,125.53,125.43$, $125.39,125.36,41.51,36.24,34.74,34.71,34.67,34.58,34.57,33.88,31.49,31.46,31.41$, 29.23, 27.84, 22.79, 20.61, 19.61, 18.93, 14.49, 11.61; HRMS-ESI (m/z) $[\mathrm{M}+\mathrm{Na}]^{+}$calcd for $\mathrm{C}_{48} \mathrm{H}_{56} \mathrm{Na} 655.4280$, found 655.4292 .

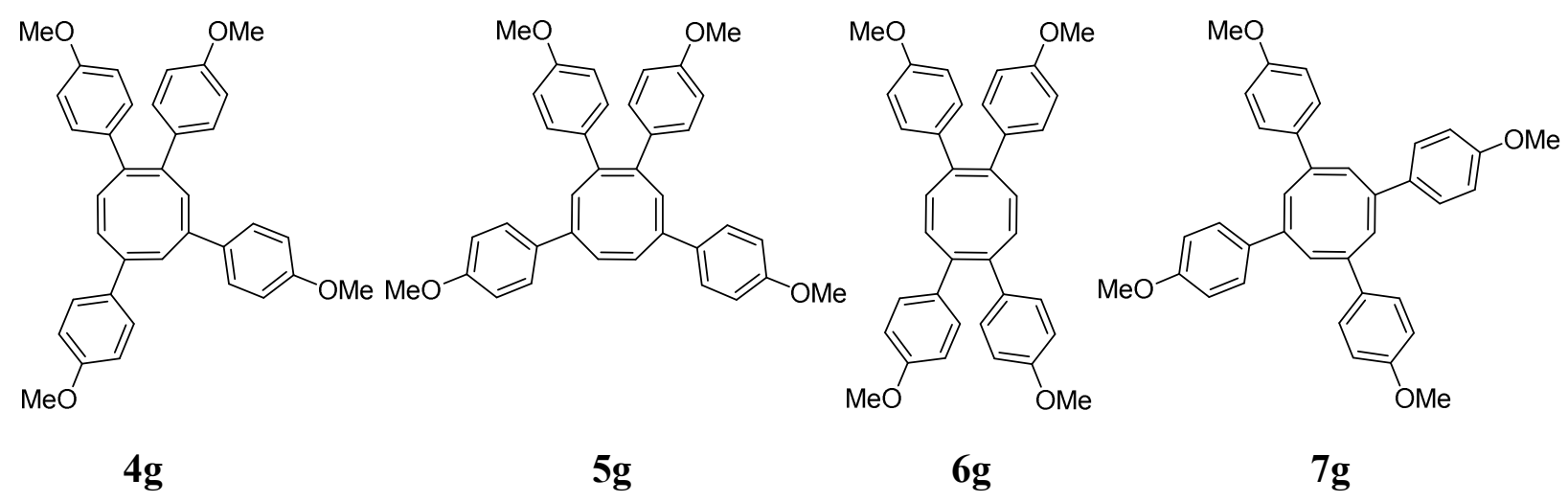

p-Anisole substituted COTs (4g, 5g, 6g (or 7g))

Purification from the benzene derivatives by silica flash column chromatography (80: 20 petroleum ether: ethyl acetate) yielded the mixture of $p$-anisole substituted COT isomers in $7 \%$ combined yield. ${ }^{1} \mathrm{H} \mathrm{NMR}\left(\mathrm{CDCl}_{3}, 400 \mathrm{MHz} \delta\right): 7.59-6.72(\mathrm{~m}, \mathrm{ArH}$ and COT hydrogens of $\mathbf{4 g}$, $\mathbf{5 g}, \mathbf{6 g}$ (or 7g)), 6.69 (dd, $J=4.0,0.8 \mathrm{~Hz}, 1 \mathrm{H}$ of $\mathbf{4 g}), 6.67-6.48$ (4s, COT hydrogens of $\mathbf{4 g}, \mathbf{5 g}, \mathbf{6 g}$ (or 7g)), 3.89-3.71 (8s, $\mathrm{OCH}_{3}$ of $\mathbf{4 g}, \mathbf{5 g}, \mathbf{6 g}$ (or 7g)); ${ }^{13} \mathrm{C}\left\{{ }^{1} \mathrm{H}\right\} \mathrm{NMR}\left(\mathrm{CDCl}_{3}, 101 \mathrm{MHz}, \delta\right.$ ) of $\mathbf{4 g}$, 5g, 6g (or 7g): 159.50, 159.35, 159.33, 159.04, 158.84, 158.82, 143.86, 142.71, 142.16, 142.04, $141.57,140.86,133.21,132.88,132.68,132.63,132.55,132.53,132.42,128.46,128.32,128.22$, $128.14,127.87,127.86,127.77,127.50,127.38,126.33,126.21,125.81,125.79,114.42,114.03$, 113.98, 113.89, 113.84, 113.82, 113.81, 113.72, 55.51, 55.48, 55.33, 55.32, 55.29; HRMS-ESI $(\mathrm{m} / \mathrm{z})\left[\mathrm{M}+\mathrm{Ag}^{107}\right]^{+}$calcd for $\mathrm{C}_{36} \mathrm{H}_{32} \mathrm{O}_{4} \mathrm{Ag}$ 635.1346, found 635.1345. 


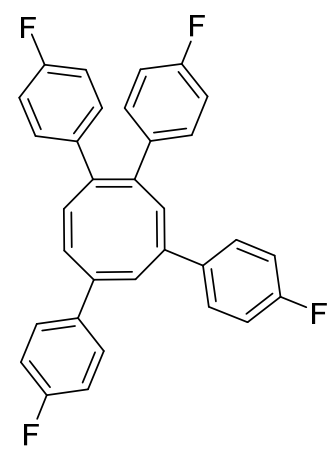

4h

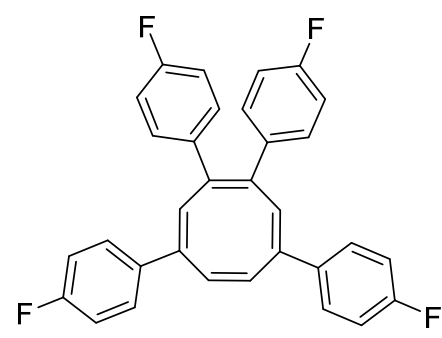

$5 \mathbf{h}$

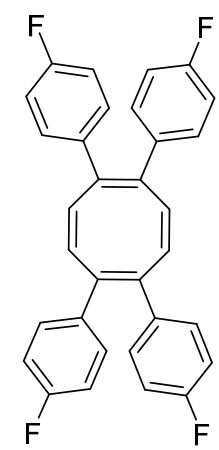

6h

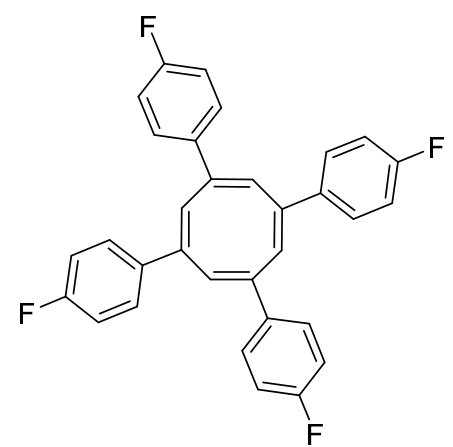

$7 \mathbf{h}$

p-Fluorophenyl substituted COTs (4h, 5h, 6h (or 7h))

Purification from the benzene derivatives by silica flash column chromatography (100\% petroleum ether) yielded the mixture of $p$-fluorophenyl substituted COT isomers in 29\% combined yield. ${ }^{1} \mathrm{H}$ NMR $\left(\mathrm{CDCl}_{3}, 400 \mathrm{MHz}, \delta\right): 7.58-6.88(\mathrm{~m}, 48 \mathrm{H}$ of $\mathbf{4 h}, \mathbf{5 h}, \mathbf{6 h}$ (or $\mathbf{7 h})$ ), 6.79 (s, 2H of 5h)), 6.77 (s, 1H of $\mathbf{4 h}), 6.73-6.72(\mathrm{~d}, J=4 \mathrm{~Hz}, 1 \mathrm{H}$ of $4 \mathbf{h}), 6.71$ (s, $4 \mathrm{H}$ of $\mathbf{6 h}$ or $7 \mathbf{h})$, 6.58 (s, $1 \mathrm{H}$ of $\mathbf{4 h}), 6.53$ (s, $1 \mathrm{H}$ of $\mathbf{4 h}), 6.52$ (s, 2H of $\mathbf{5 h}) ;{ }^{13} \mathrm{C}\left\{{ }^{1} \mathrm{H}\right\} \mathrm{NMR}\left(\mathrm{CDCl}_{3}, 101 \mathrm{MHz}, \delta\right)$ of 4h, 5h, 6h (or 7h): 162.87 (d, $\left.J_{\mathrm{CF}}=249.47 \mathrm{~Hz}\right), 162.75$ (d, $\left.J_{\mathrm{CF}}=249.47 \mathrm{~Hz}\right), 162.73$ (d, $J_{\mathrm{CF}}=$ $248.46 \mathrm{~Hz}), 162.49\left(\mathrm{~d}, J_{\mathrm{CF}}=248.46 \mathrm{~Hz}\right), 162.32\left(\mathrm{~d}, J_{\mathrm{CF}}=248.46 \mathrm{~Hz}\right), 143.72,142.36,142.04$, 141.66, 141.25, 140.46, $136.12\left(\mathrm{~d}, J_{\mathrm{CF}}=2.3 \mathrm{~Hz}\right), 135.84\left(\mathrm{~d}, J_{\mathrm{CF}}=2.7 \mathrm{~Hz}\right), 135.64\left(\mathrm{~d}, J_{\mathrm{CF}}=3.3\right.$ $\mathrm{Hz}), 135.45\left(\mathrm{~d}, J_{\mathrm{CF}}=3.2 \mathrm{~Hz}\right), 135.32\left(\mathrm{~d}, J_{\mathrm{CF}}=3.2 \mathrm{~Hz}\right), 130.03\left(\mathrm{~d}, J_{\mathrm{CF}}=1.1 \mathrm{~Hz}\right), 129.94\left(\mathrm{~d}, J_{\mathrm{CF}}=\right.$ $1.0 \mathrm{~Hz}), 129.57\left(\mathrm{~d}, J_{\mathrm{CF}}=1.0 \mathrm{~Hz}\right), 127.04\left(\mathrm{~d}, J_{\mathrm{CF}}=1.0 \mathrm{~Hz}\right)$, the rest of the resonances are not assigned due to the complication of fluorine coupling; HRMS-ESI (m/z) $\left[\mathrm{M}+\mathrm{Ag}^{107}\right]^{+}$calcd for $\mathrm{C}_{32} \mathrm{H}_{20} \mathrm{~F}_{4} \mathrm{Ag}$ 587.0547, found 587.0548.

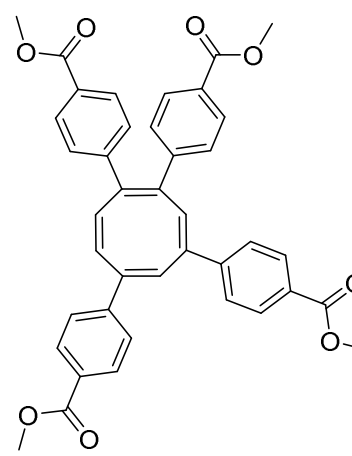

$4 \mathbf{i}$

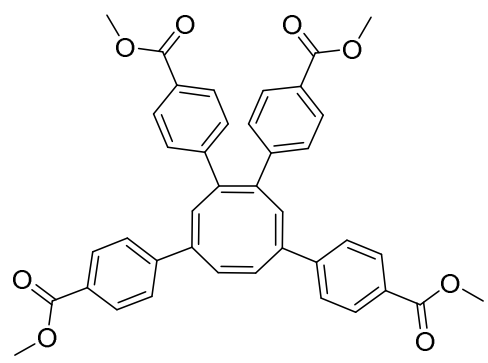

$5 \mathbf{i}$

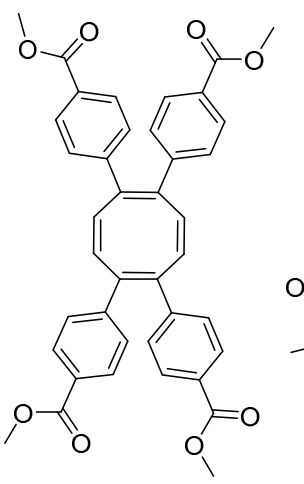

6i

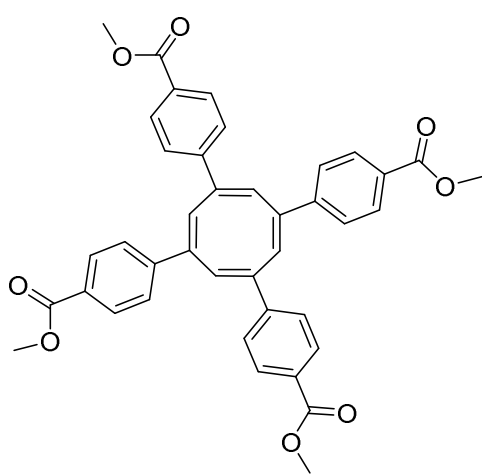

$7 \mathbf{i}$

\section{p-Methyl benzoate substituted COTs (4i, 5i, 6i (or 7i))}

Purification from the benzene derivatives by silica flash column chromatography (100\% petroleum ether) yielded the mixture of $p$-methyl benzoate substituted COT isomers in $40 \%$ combined yield. ${ }^{1} \mathrm{H} \mathrm{NMR}\left(\mathrm{CDCl}_{3}, 500 \mathrm{MHz}, \delta\right): 8.11-7.40$ (m, $\mathrm{ArH}$ of 4i, 5i, 6i (or 7i)), 7.03 (s, 
1H of $4 \mathbf{i})$ ), 7.01 (s, 2H of $\mathbf{5 i}), 6.95$ (br, $1 \mathrm{H}$ of $4 \mathbf{i}), 6.94$ (s, $4 \mathrm{H}$ of $\mathbf{6 i}$ or $7 \mathbf{i}), 6.80$ (s, 1H of $4 \mathbf{i}), 6.76$ (s, $2 \mathrm{H}$ of $\mathbf{5 i}), 6.75$ (s, $1 \mathrm{H}$ of $\mathbf{4 i}), 3.96-3.85\left(\mathrm{~m}, \mathrm{CH}_{3}\right.$ of $\mathbf{4 i}, \mathbf{5 i}, \mathbf{6} \mathbf{i}($ or $7 \mathbf{i}) ;{ }^{13} \mathrm{C}\left\{{ }^{1} \mathrm{H}\right\} \mathrm{NMR}\left(\mathrm{CDCl}_{3}\right.$, $126 \mathrm{MHz}, \delta$ ) of $\mathbf{4 i}, \mathbf{5 i}, \mathbf{6 i}$ (or 7i): 166.88, 166.85, 166.74, 166.70, 130.25, 130.20, 130.13, 130.04, $129.95,129.72,129.62,127.22,127.05,126.64,126.54,126.16,126.08,52.34,52.24$; HRMSESI $(\mathrm{m} / \mathrm{z})[\mathrm{M}+\mathrm{Na}]^{+}$calcd for $\mathrm{C}_{40} \mathrm{H}_{32} \mathrm{O}_{8} \mathrm{Na} 663.1989$, found 663.1992 . 


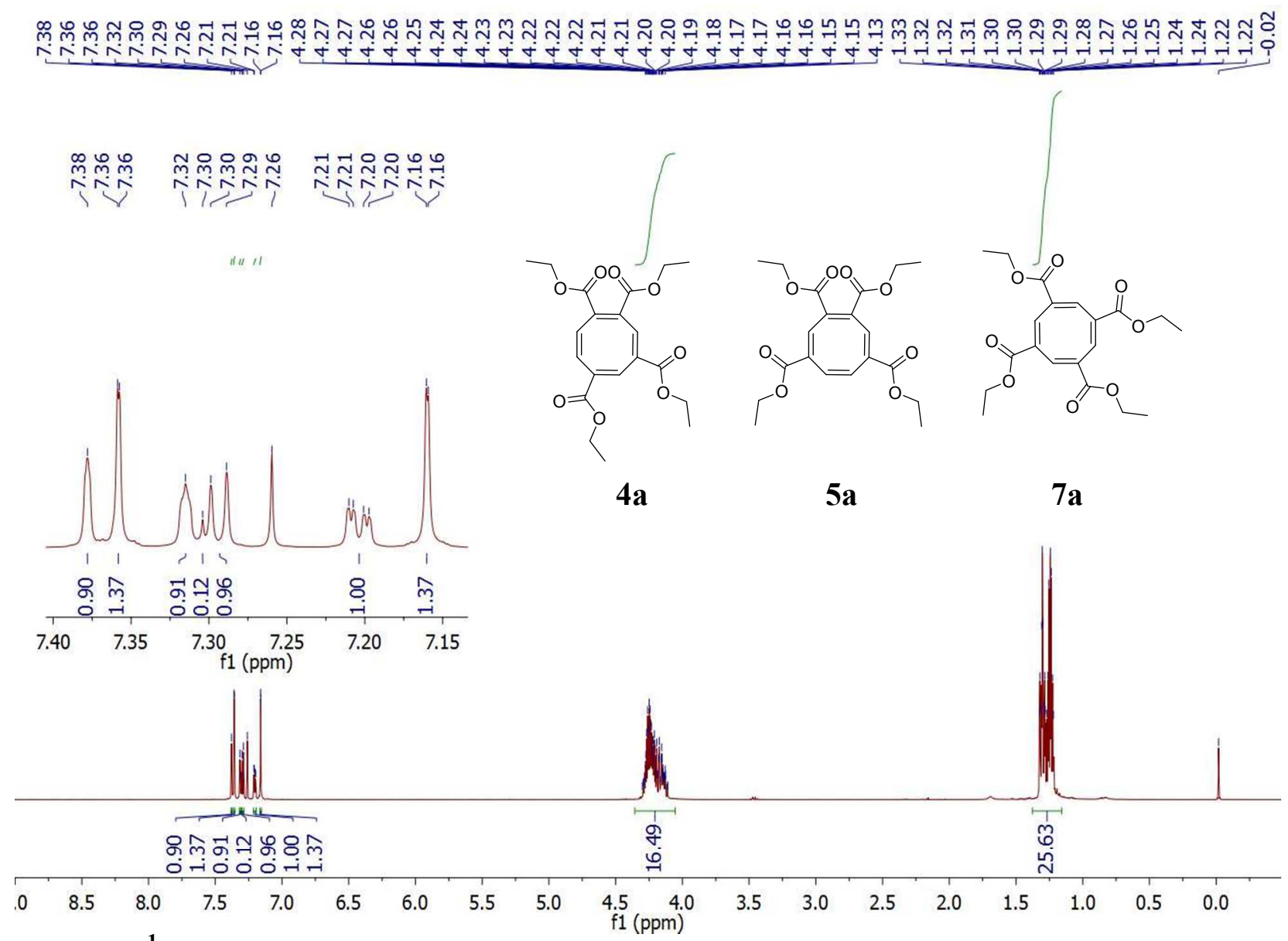

Figure 1. ${ }^{1} \mathrm{H}$ NMR spectrum of ethyl esters (4a, 5a, 7a). 


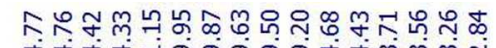

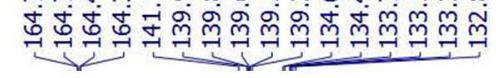

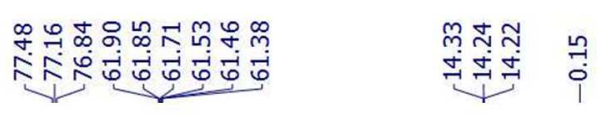

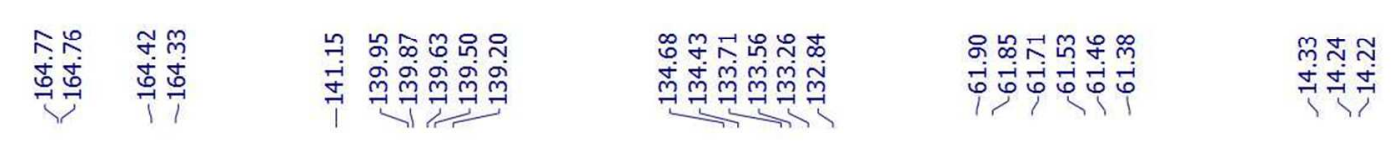

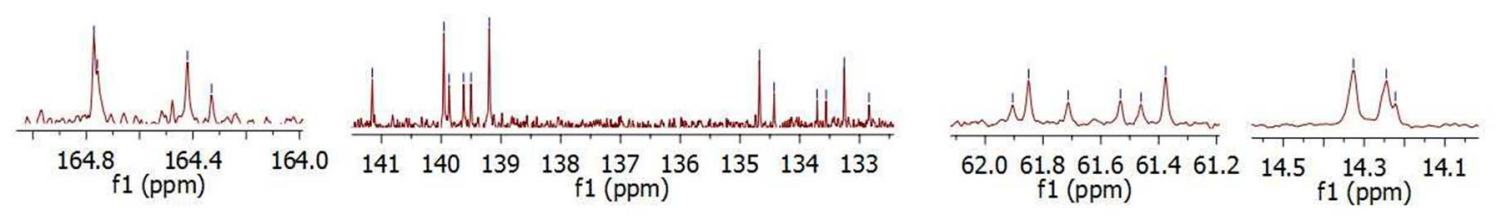

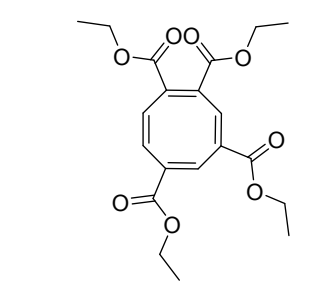

$4 \mathbf{a}$

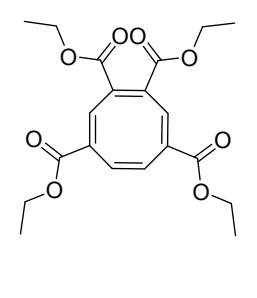

$5 \mathbf{a}$

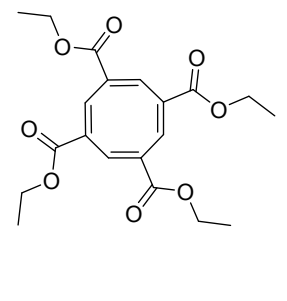

$7 \mathbf{a}$

\begin{tabular}{|c|c|c|c|c|c|c|c|c|c|c|c|c|c|c|c|c|c|c|c|c|c|c|}
\hline & & & & & & & -18 & & & & & & & & l & & & & & & 1 & \\
\hline 210 & 200 & 190 & 180 & 170 & 160 & 150 & 140 & 130 & 120 & 110 & $\begin{array}{l}100 \\
1 \mathrm{ppm}\end{array}$ & 90 & 80 & 70 & 60 & 50 & 40 & 30 & 20 & 10 & 0 & -10 \\
\hline
\end{tabular}

Figure 2. ${ }^{13} \mathrm{C}\left\{{ }^{1} \mathrm{H}\right\}$ NMR spectrum of ethyl esters (4a, 5a, 7a). 


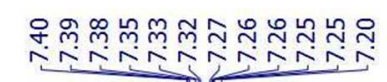

|| II
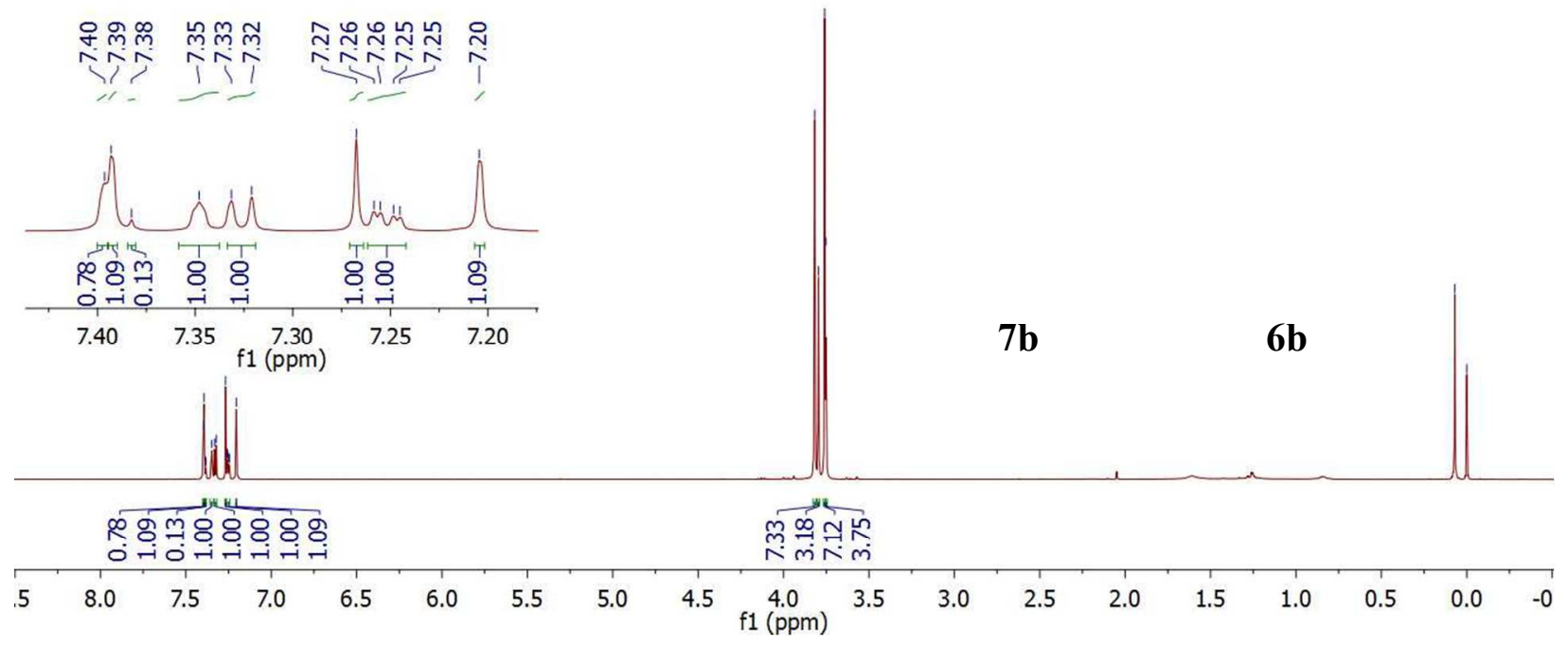

Figure 3. ${ }^{1} \mathrm{H}$ NMR spectrum of methyl esters (4b-7b).<smiles>COC(=O)c1cc(C(=O)OC)cc2c(OC)c(=O)oc-2c1</smiles><smiles>COC(=O)C1=C(C(=O)OC)C=CC(C(=O)OC)=C(C(=O)OC)C=C1</smiles><smiles>COC(=O)C1=CC(C(=O)OC)=CC(C(=O)OC)=CC(C(=O)OC)=C1</smiles><smiles>COC(=O)C1=CC=C(C(=O)OC)C=C2C(OC)OC(OC)C2C(OC)=C1</smiles> 


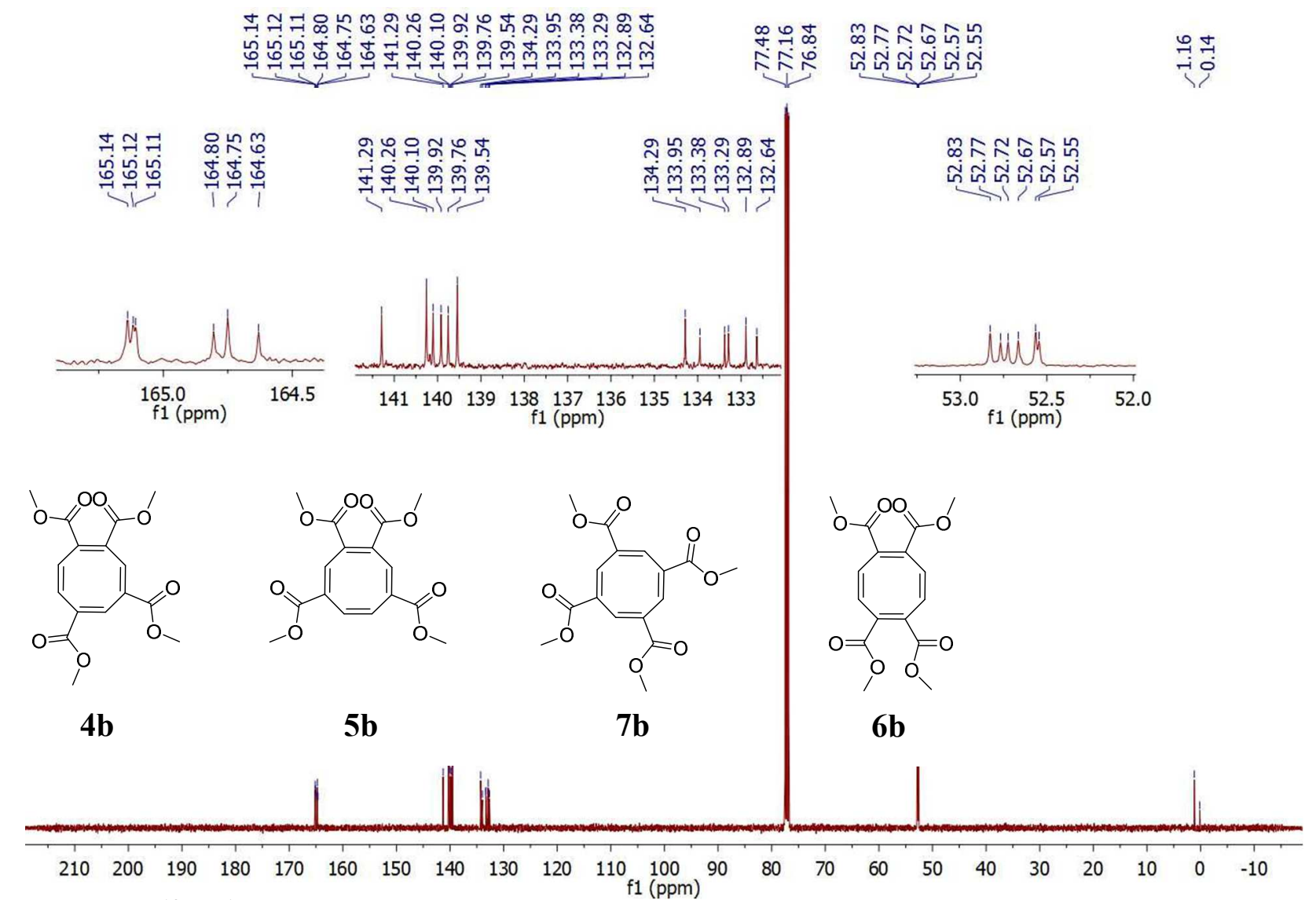

Figure 4. ${ }^{13} \mathrm{C}\left\{{ }^{1} \mathrm{H}\right\}$ NMR spectrum of methyl esters (4b-7b). 


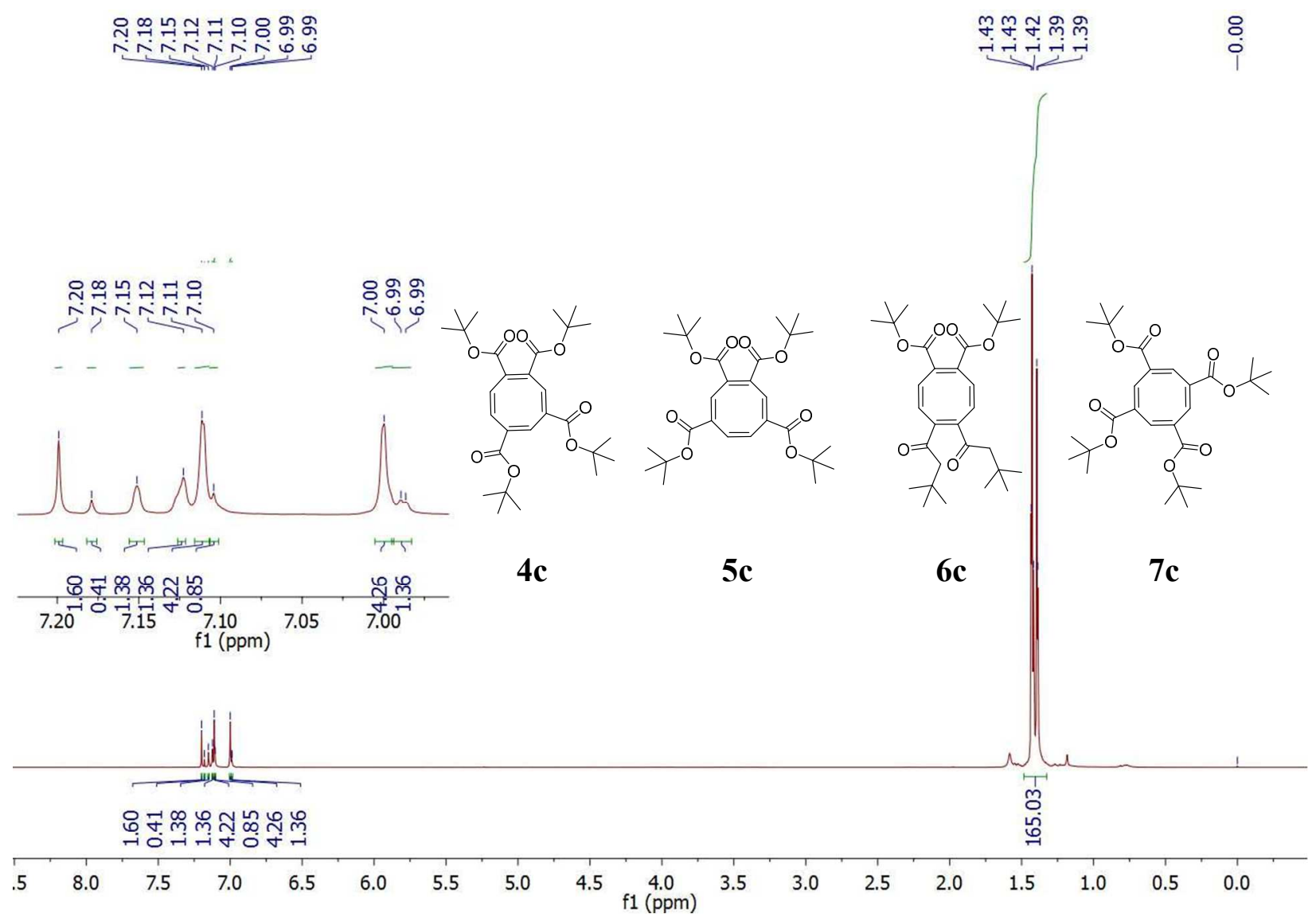

Figure 5. ${ }^{1} \mathrm{H}$ NMR spectrum of $t$-butyl esters (4c-7c). 


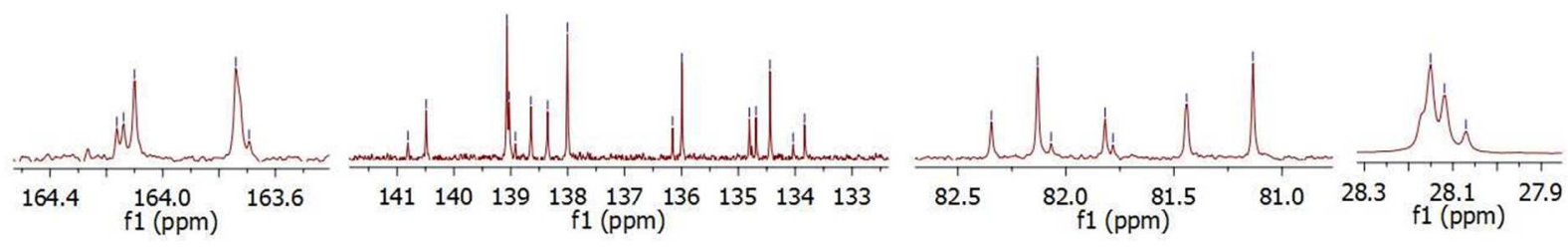

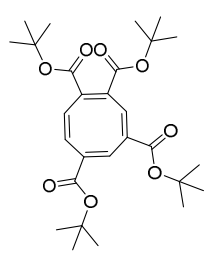
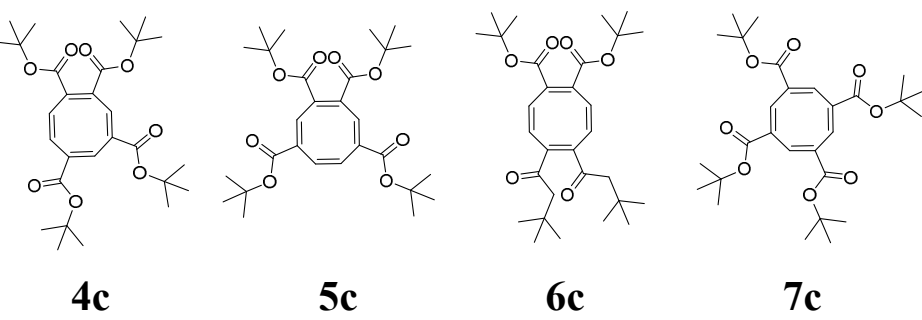

$5 c$

6c

7c

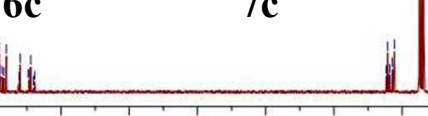

$\begin{array}{llllll}210 & 200 & 190 & 180 & 170 & 160\end{array}$

$$
\text { f1 (ppm) }
$$

Figure 6. ${ }^{13} \mathrm{C}\left\{{ }^{1} \mathrm{H}\right\}$ NMR spectrum of $t$-butyl esters (4c-7c). 


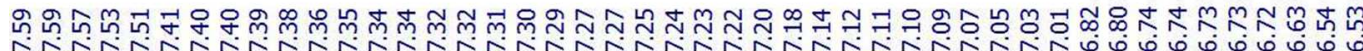
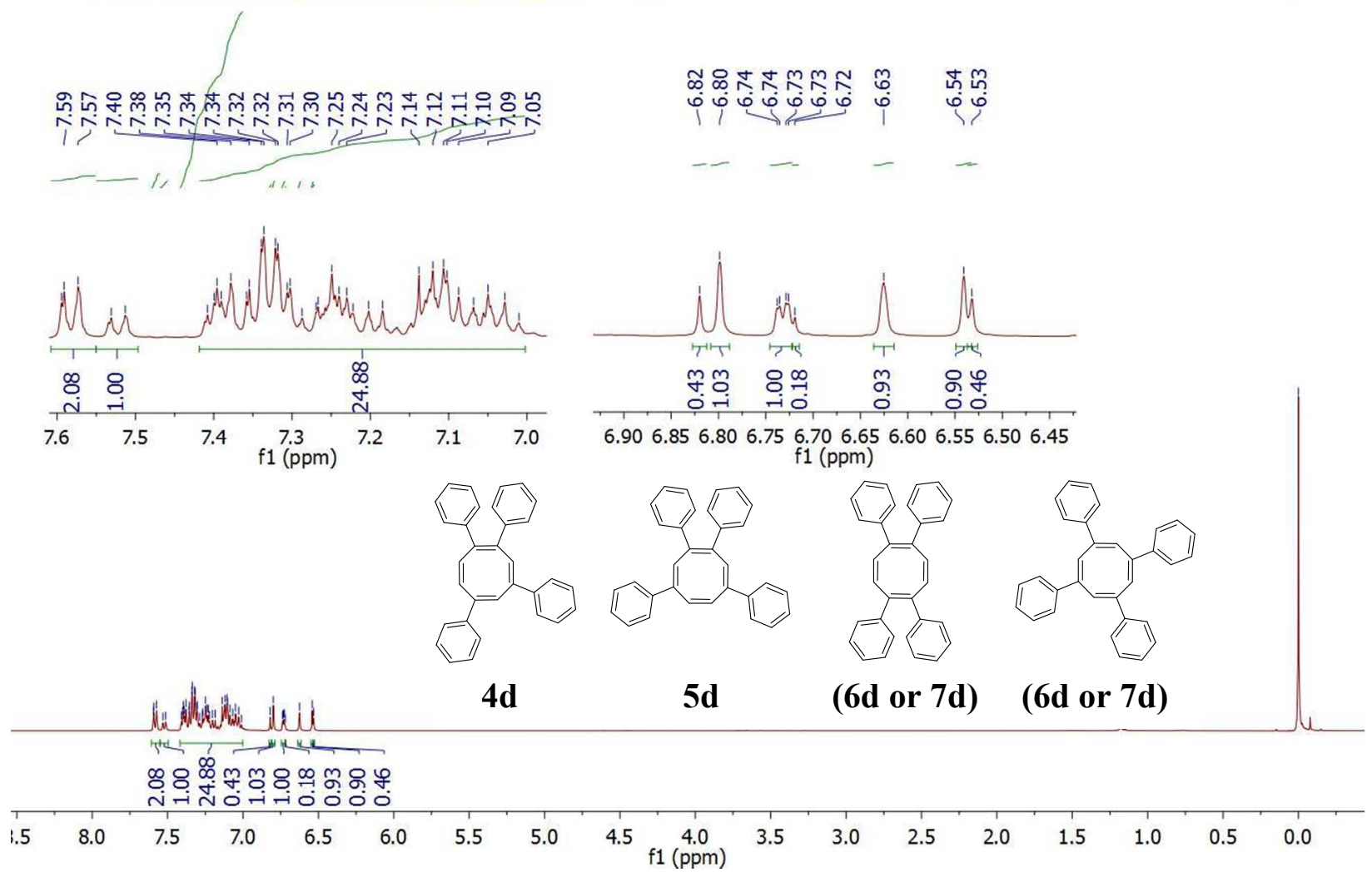

Figure 7. ${ }^{1} \mathrm{H}$ NMR spectrum of phenyl substituted COTs (4d, 5d, 6d (or 7d)). 

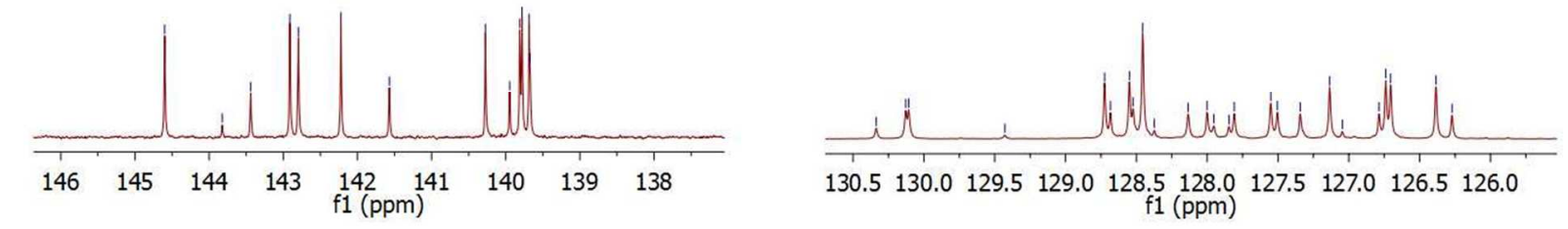

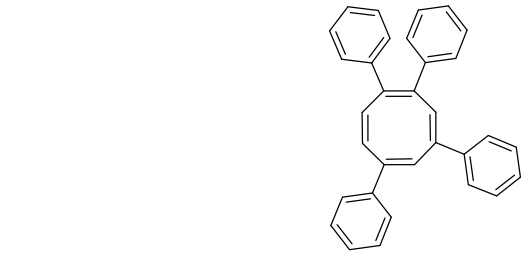

4d

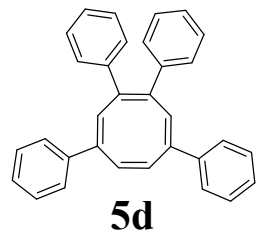

$5 d$

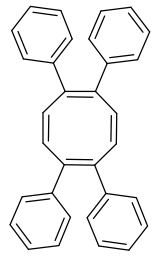

(6d or 7d)

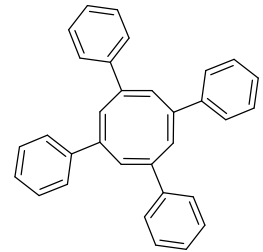

(6d or 7d)

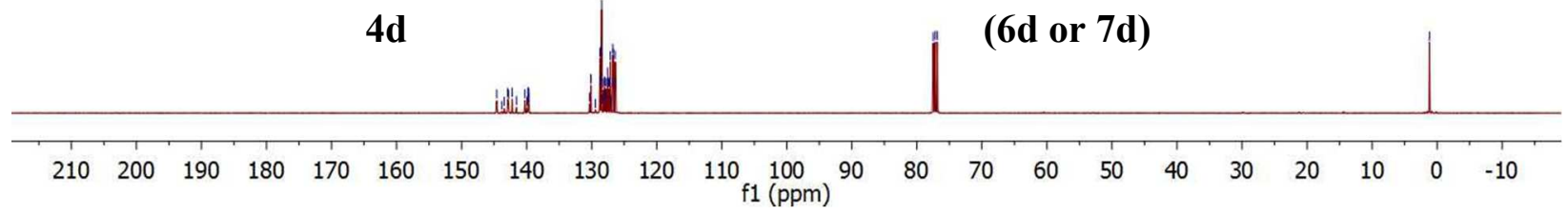

Figure 8. ${ }^{13} \mathrm{C}\left\{{ }^{1} \mathrm{H}\right\}$ NMR spectrum of phenyl substituted COTs (4d, 5d, 6d (or 7d)). 

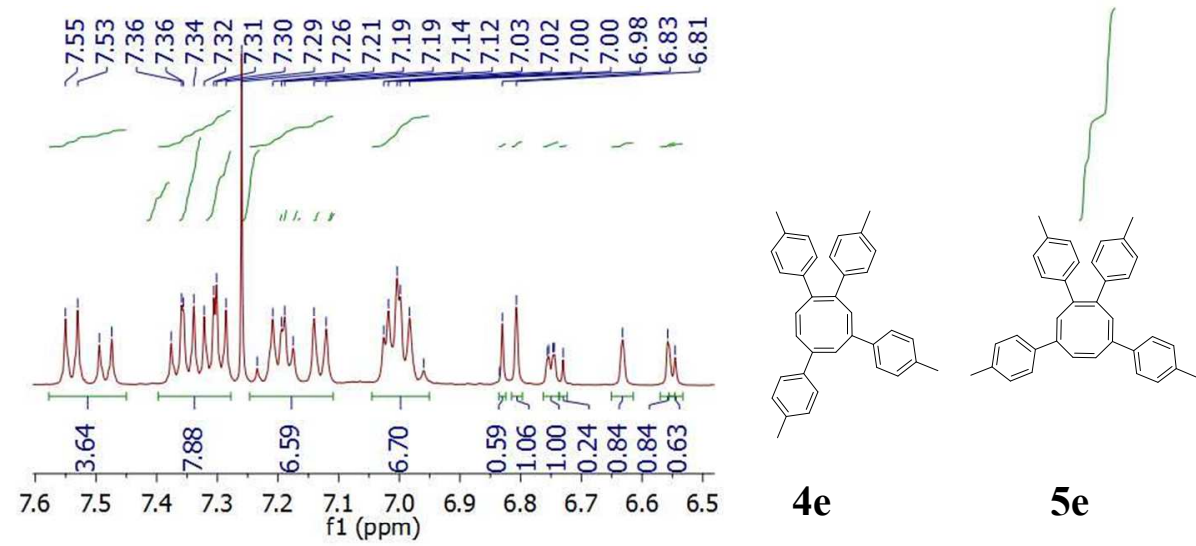

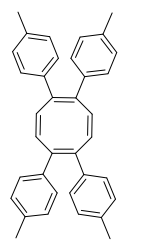

5 e

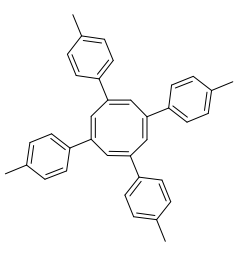

(6e or $7 e) \quad(6 e$ or $7 e)$

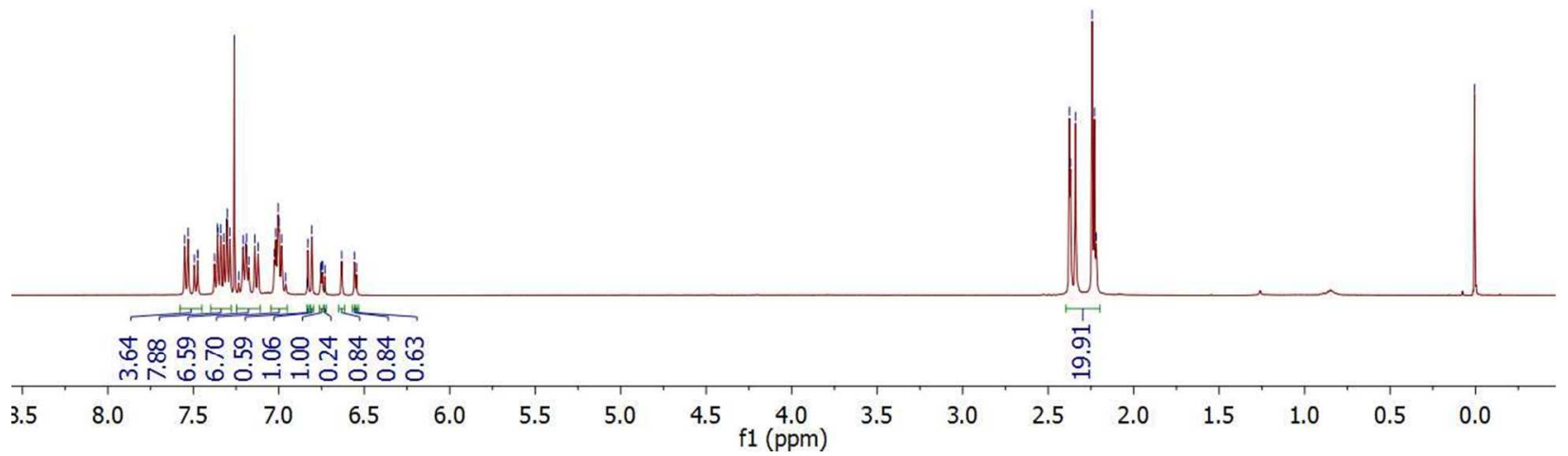

Figure 9. ${ }^{1} \mathrm{H}$ NMR spectrum of $p$-tolyl substituted COTs (4e, 5e, 6e (or 7e)). 


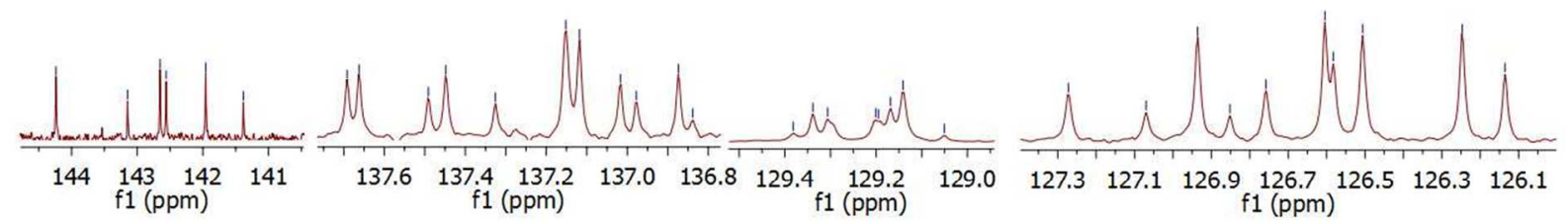

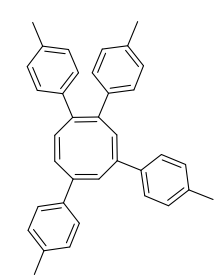

$4 \mathrm{e}$

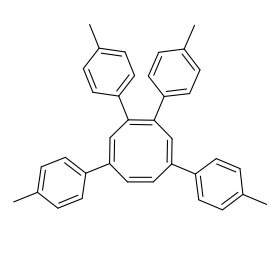

$5 e$

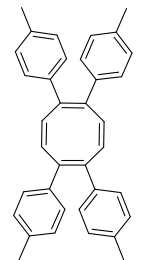

(6e or 7e)

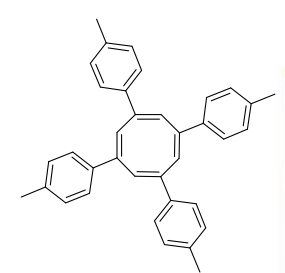

(6e or 7e)

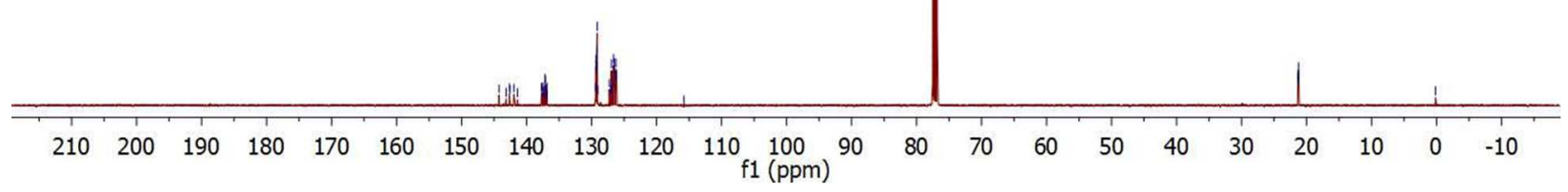

Figure 10. ${ }^{13} \mathrm{C}\left\{{ }^{1} \mathrm{H}\right\}$ NMR spectrum of $p$-tolyl substituted COTs (4e, 5e, 6e (or 7e)). 


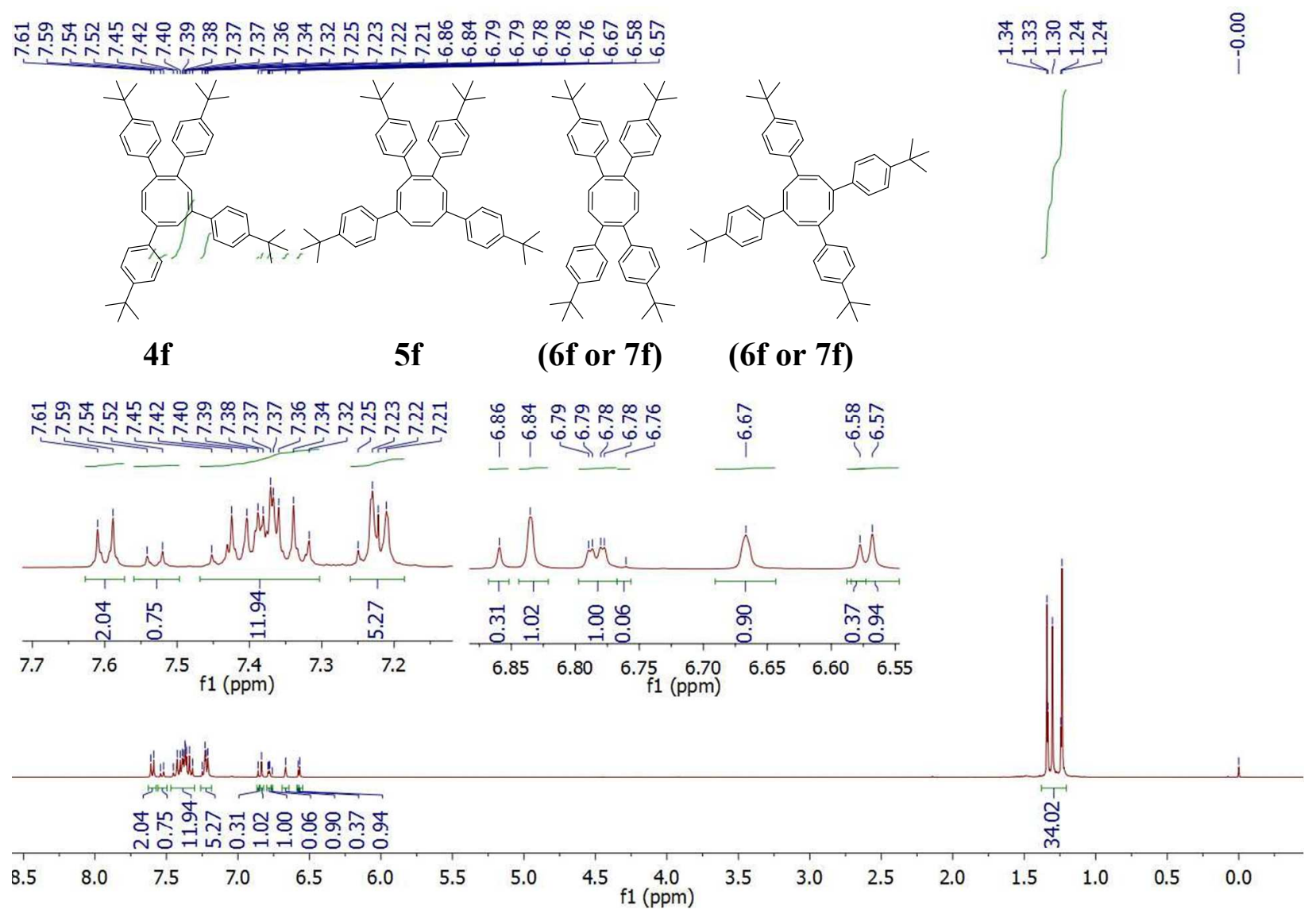

Figure 11. ${ }^{1} \mathrm{H}$ NMR spectrum of $p$-tert-butylphenyl substituted COTs (4f, 5f, $6 \mathrm{f}$ (or $\left.7 \mathrm{f}\right)$ ). 


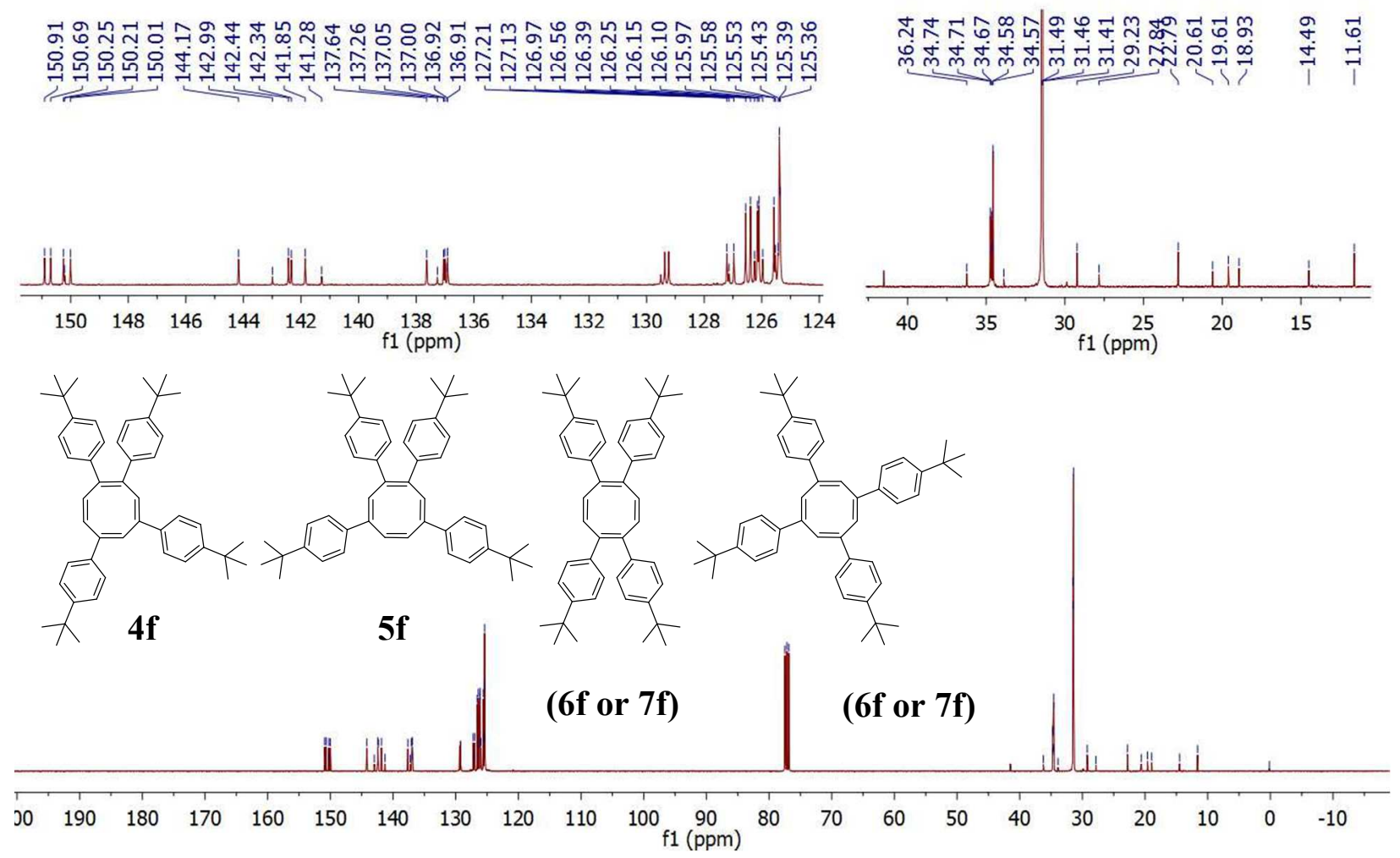

Figure 12. ${ }^{13} \mathrm{C}\left\{{ }^{1} \mathrm{H}\right\}$ NMR spectrum of $p$-tert-butylphenyl substituted COTs (4f, 5f, $6 \mathrm{f}$ (or 7f)) . 

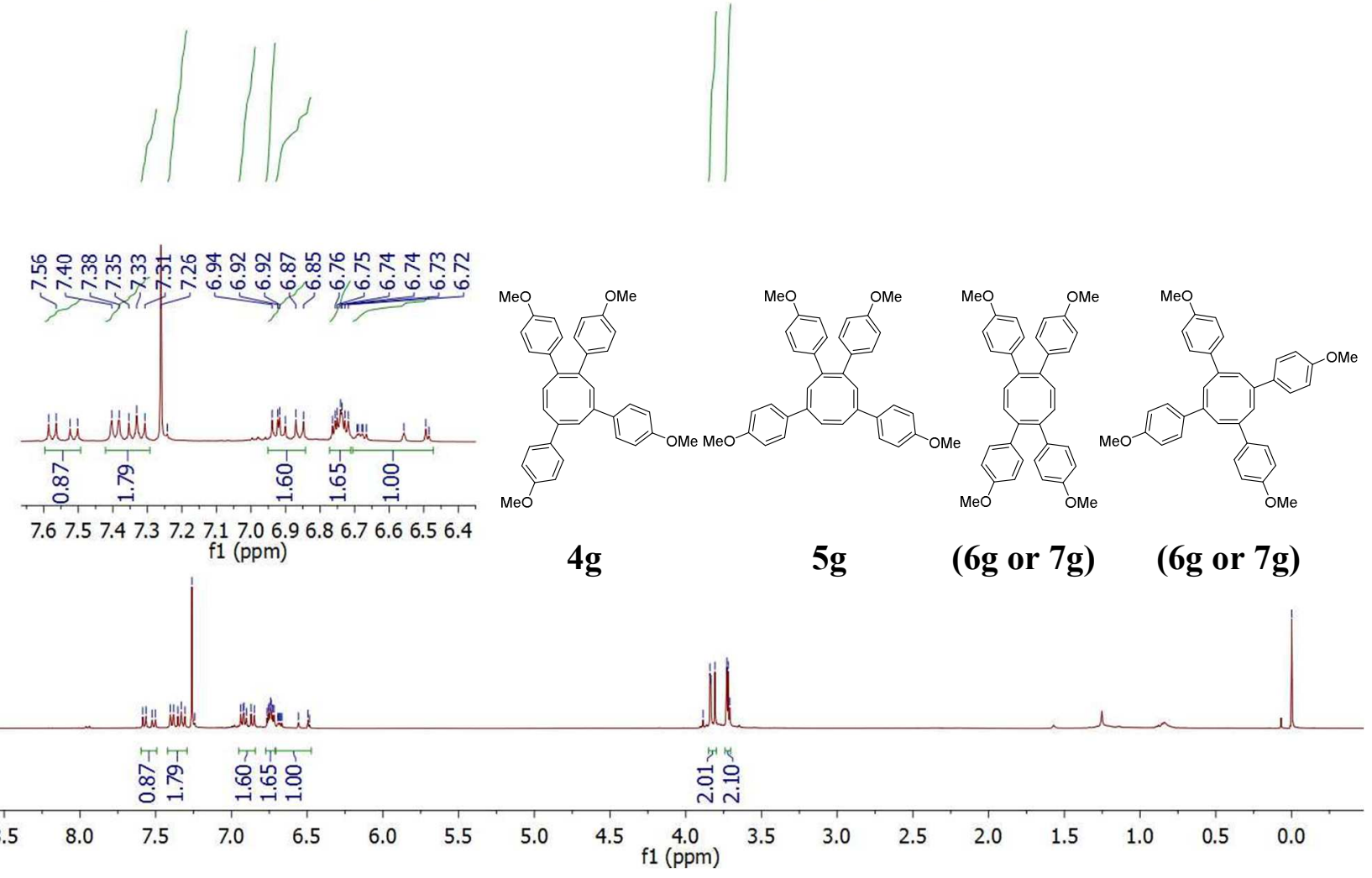

Figure 13. ${ }^{1} \mathrm{H}$ NMR spectrum of $p$-anisole substituted COTs (4g, 5g, $6 \mathrm{~g}(\mathrm{or} 7 \mathrm{~g})$ ). 


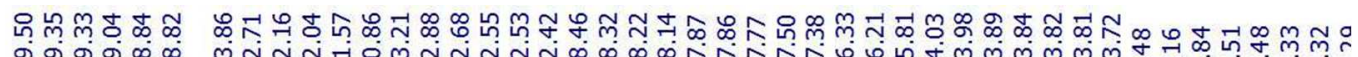

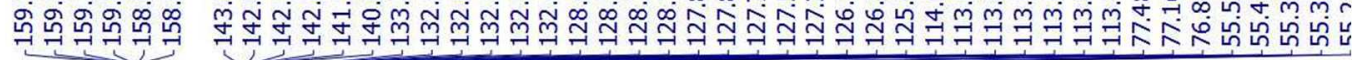

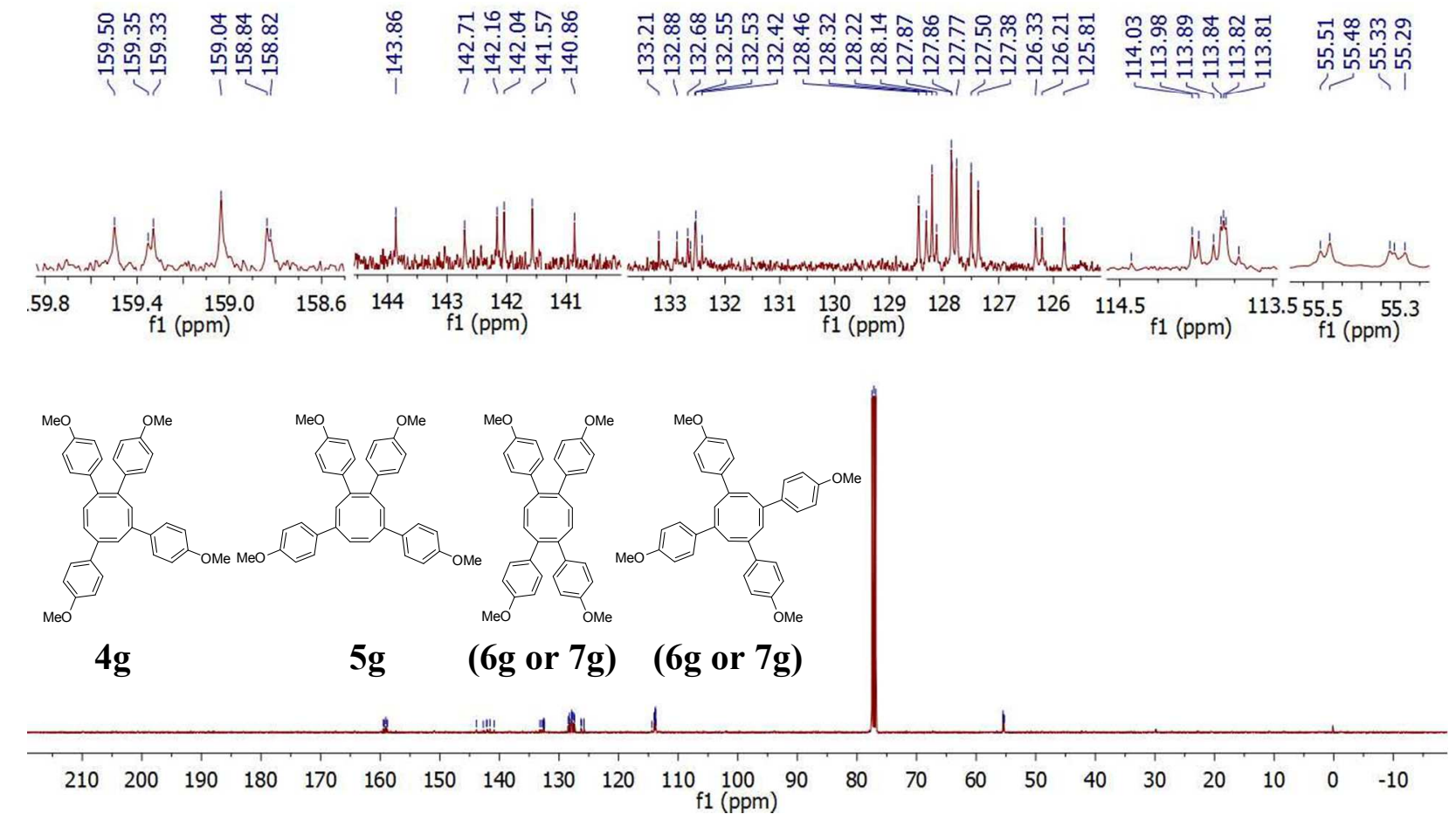

Figure 14. ${ }^{13} \mathrm{C}\left\{{ }^{1} \mathrm{H}\right\}$ NMR spectrum of $p$-anisole substituted COTs $(4 \mathrm{~g}, 5 \mathrm{~g}, 6 \mathrm{~g}$ (or $\left.7 \mathrm{~g})\right)$. 


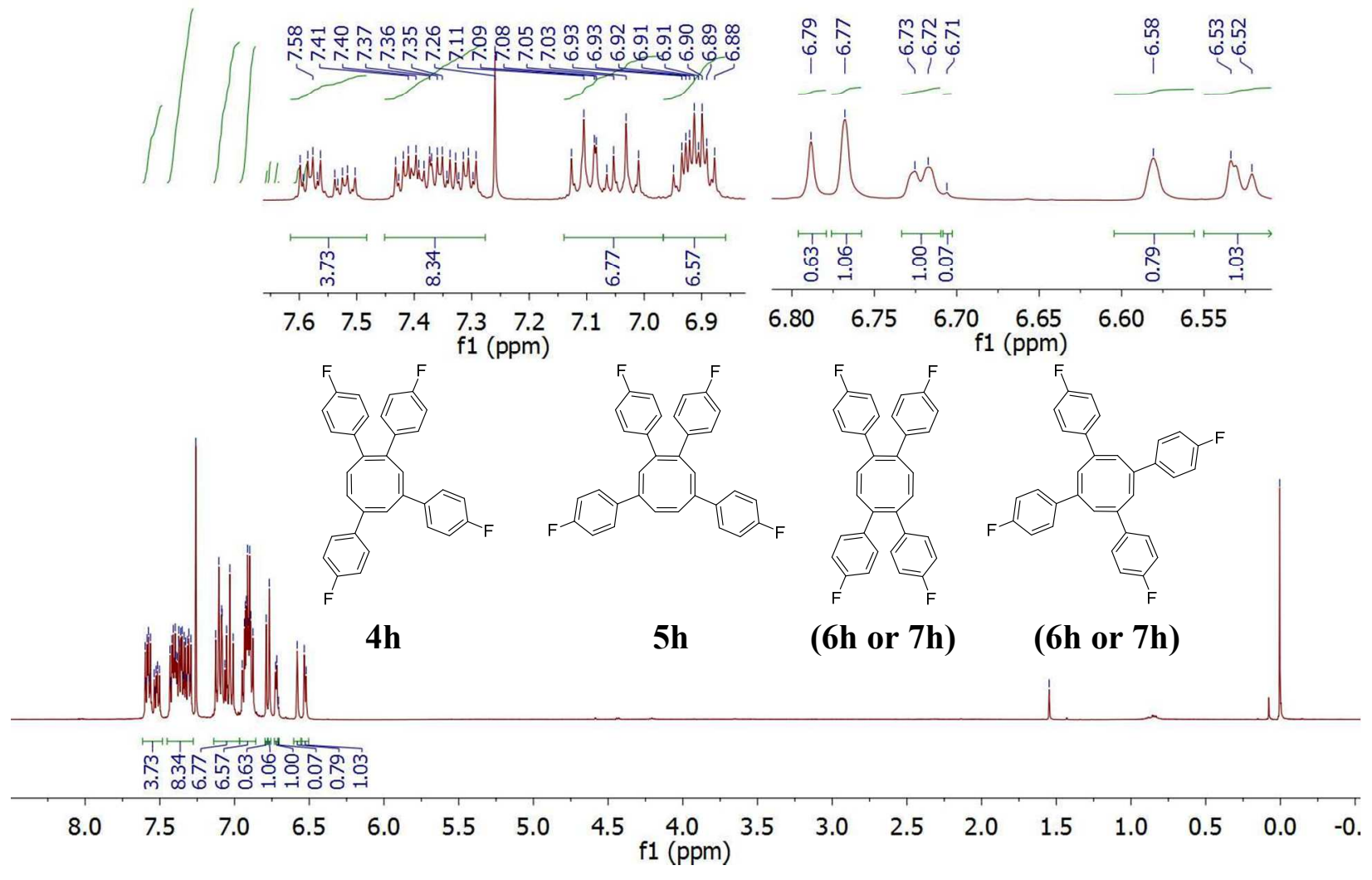

Figure 15. ${ }^{1} \mathrm{H}$ NMR spectrum of $p$-fluorophenyl substituted COTs $(4 \mathrm{~h}, 5 \mathrm{~h}, 6 \mathrm{~h}$ (or $7 \mathrm{~h})$ ). 


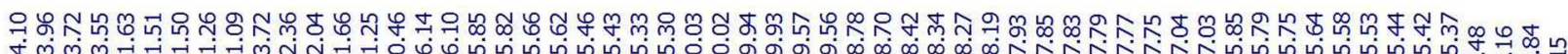

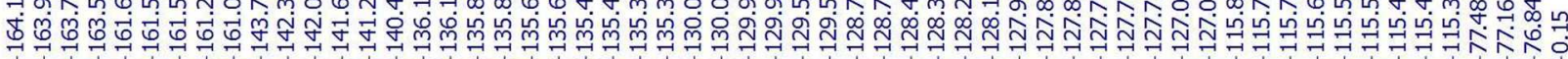

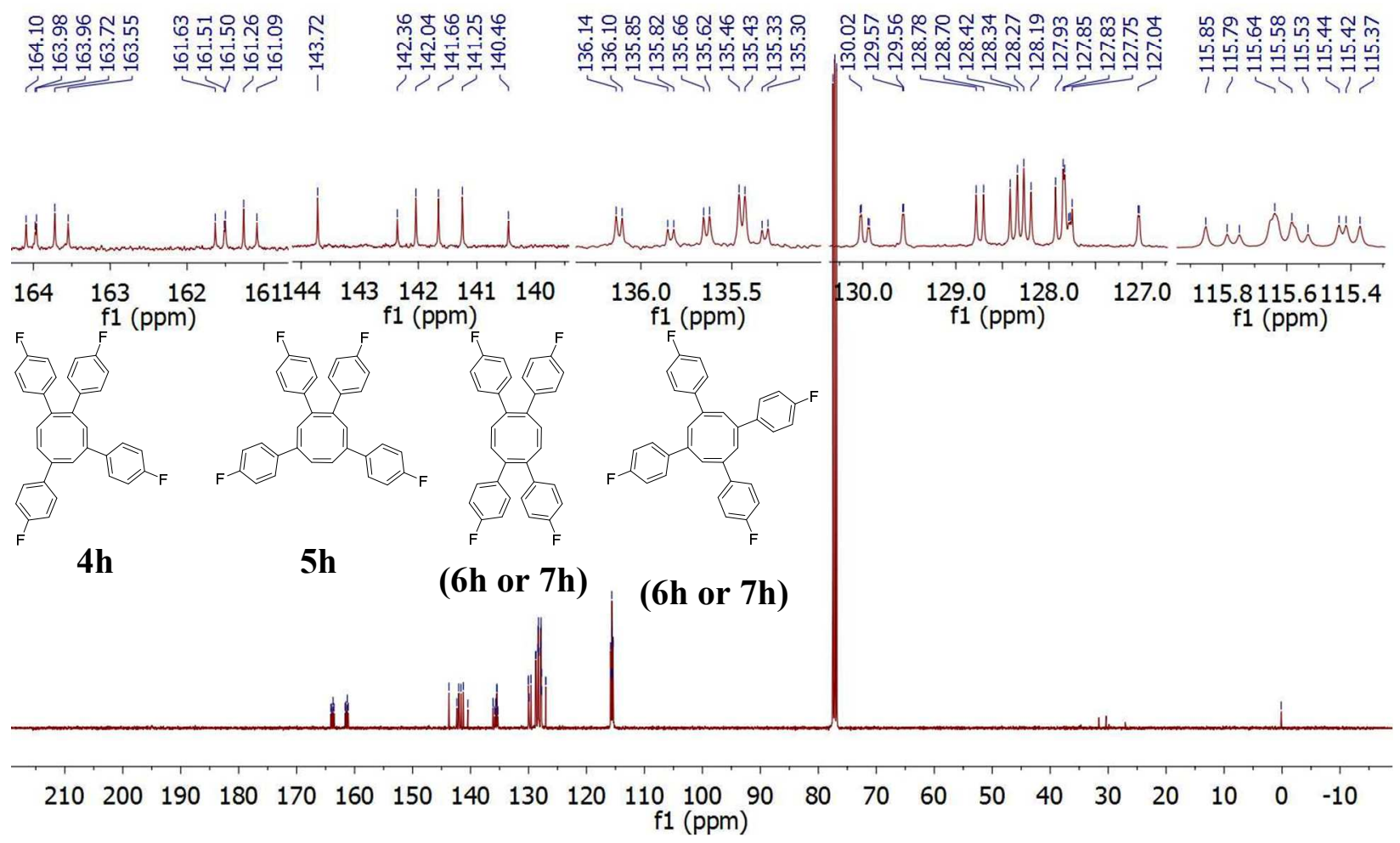

Figure 16. ${ }^{13} \mathrm{C}\left\{{ }^{1} \mathrm{H}\right\}$ NMR spectrum of $p$-fluorophenyl substituted COTs $(4 \mathrm{~h}, 5 \mathrm{~h}, 6 \mathrm{~h}$ (or $\left.7 \mathrm{~h})\right)$. 


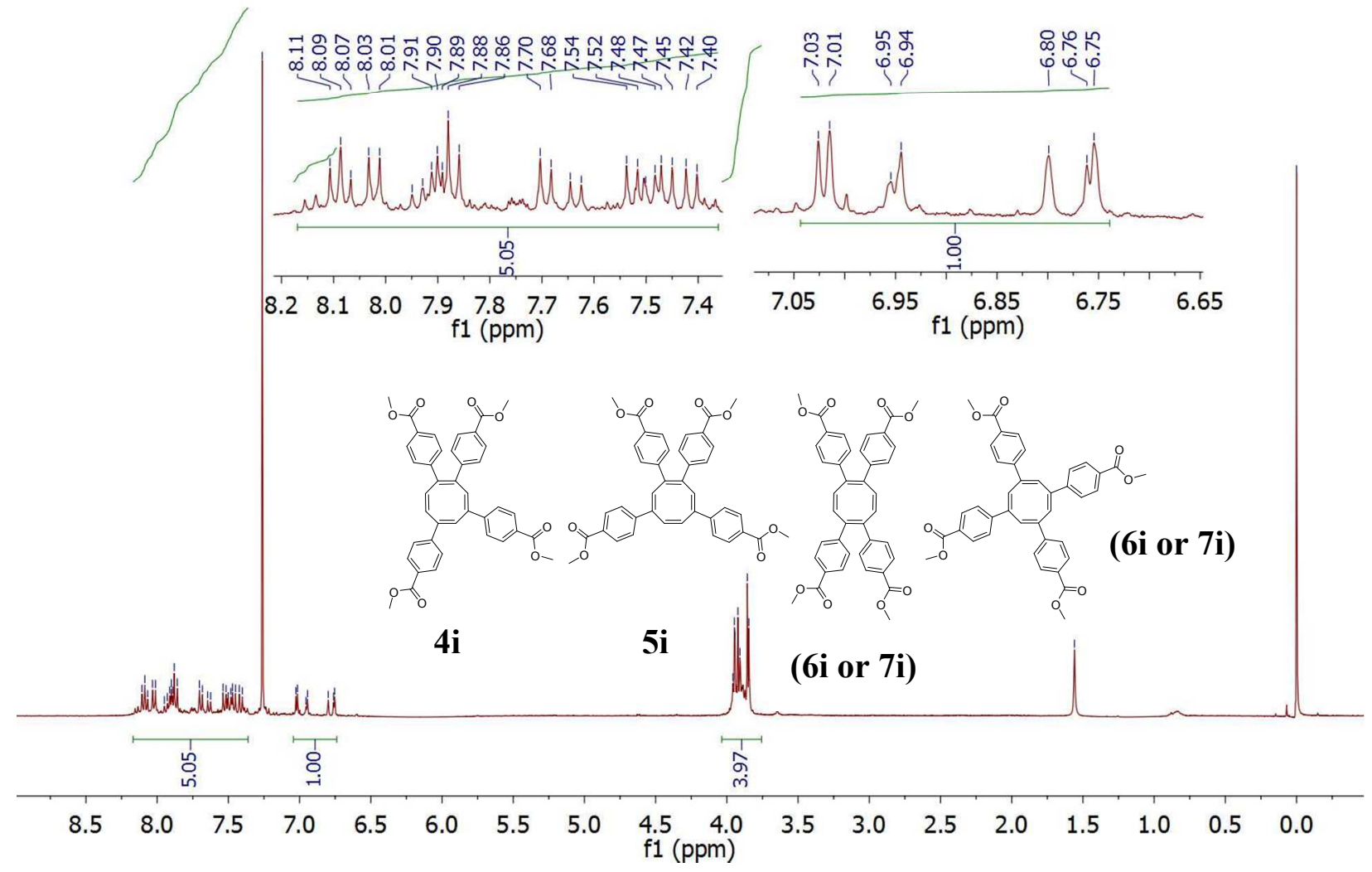

Figure 17. ${ }^{1} \mathrm{H}$ NMR spectrum of $p$-methyl benzoate substituted COTs (4i, 5i, 6i (or 7i)). 


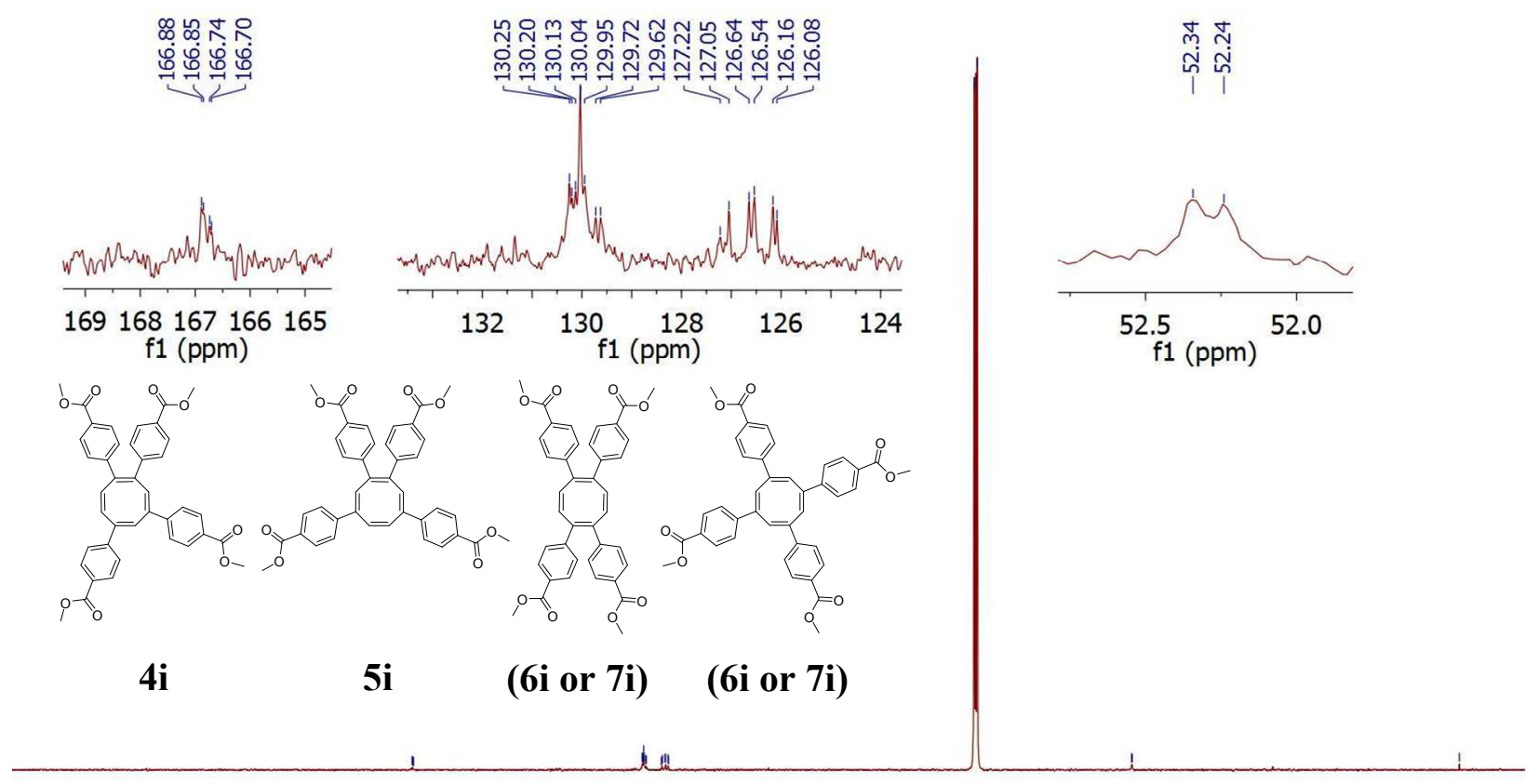

$\begin{array}{llllllllllllllllllllllllllll}30 & 220 & 210 & 200 & 190 & 180 & 170 & 160 & 150 & 140 & 130 & 120 & 110 & 100 & 90 & 80 & 70 & 60 & 50 & 40 & 30 & 20 & 10 & 0 & -1\end{array}$

Figure 18. ${ }^{13} \mathrm{C}\left\{{ }^{1} \mathrm{H}\right\}$ NMR spectrum of $p$-methyl benzoate substituted COTs (4i, 5i, 6i (or 7i)). 


\section{References:}

1. Rodrigo, S. K.; Powell, I. V.; Coleman, M. G.; Krause, J. A.; Guan, H. Efficient and regioselective nickel-catalyzed [2+2+2] cyclotrimerization of ynoates and related alkynes. Org. Biomol. Chem. 2013, 11, 7653-7657.

2. Xu, L.; Yu, R.; Wang, Y.; Chen, J; Yang, Z. Highly Regioselective Synthesis of Substituted Triphenylenes from 1,2,4-Trisubstituted Arenes via a Co-Catalyzed Intermolecular Alkyne Cyclotrimerization. J. Org. Chem. 2013, 78, 5744-5750.

3. Rassadin, V. A.; Nicolas, E.; Six, Y. Ti(OiPr) $)_{4} / n$ BuLi: an attractive reagent system for [2+2+2] cyclotrimerisation reactions. Chem. Commun. 2014, 50, 7666-7669.

4. Cadierno, V.; García-Garrido, S. E.; Gimeno, J. Efficient Intermolecular [2+2+2] Alkyne Cyclotrimerization in Aqueous Medium Using a Ruthenium(IV) Precatalyst. J. Am. Chem. Soc. 2006, 128, 15094-15095.

5. Dimroth, K.; Tüncher, W.; Kaletsch, H. Bildung und Konstitutionsaufklärung des thermischen Dimerisierungsproduktes aus 2,4,6-Tris(4-tert-butylphenyl)-phenoxyl. Chemische Berichte 1978, $111,264-271$. 


\section{X-ray Crystallography for 5a:}

Colorless thick plate-shaped crystals of $\mathbf{5 a}$ were grown from cyclohexane. For Xray examination and data collection, a suitable crystal, approximate dimensions $0.17 \mathrm{x} 0.16 \mathrm{x}$ $0.07 \mathrm{~mm}$, was mounted in a loop with Paratone-N oil and transferred to the goniostat bathed in a cold stream.

Intensity data were collected at $150 \mathrm{~K}$ on a Bruker D8 APEX-II CCD diffractometer using graphite-monochromated Mo K $\alpha$ radiation, $\lambda=0.71073 \AA$. For data collection frames were measured for a duration of 8-s with $0.3^{\circ}$ intervals of $\omega$ out to a maximum $2 \theta$ value of $\sim 56^{\circ}$. The data frames were processed using the program SAINT. The data were corrected for decay, Lorentz and polarization effects as well as absorption and beam corrections based on the multiscan technique.

The structure was solved by a combination of direct methods in SHELXTL and the difference Fourier technique and refined by full-matrix least squares on $\mathrm{F}^{2}$. Non-hydrogen atoms were refined with anisotropic displacement parameters. One ester group is disordered. A twocomponent model of the disorder did not yield a better refinement and was not pursued further. Hydrogen atoms were calculated and treated with a riding model. The $\mathrm{H}$-atom isotropic displacement parameters were defined as $\mathrm{a}^{*} \mathrm{U}_{\text {eq }}$ of the adjacent atom $(\mathrm{a}=1.5$ for methyl and 1.2 for all others.

\section{Acknowledgements, References and Notes:}

(1) Funding for the diffractometer was through NSF-MRI grant CHE-0215950.

(2) APEX2 v2014.5.0 and SAINT v8.34A data collection and data processing programs, respectively. Bruker Analytical X-ray Instruments, Inc., Madison, WI; SADABS v2014.2 semi- 
empirical absorption and beam correction program. G.M. Sheldrick, University of Göttingen, Germany; SHELXL-2014/7 \& SHELXTL v6.14 for structure solution, figures and tables, neutral-atom scattering factors as stored in this package. G.M. Sheldrick, University of Göttingen, Germany and Bruker Analytical X-ray Instruments, Inc., Madison, WI. 
Table S1. Crystal data and structure refinement for $\mathrm{C}_{20} \mathrm{H}_{24} \mathrm{O}_{8}(\mathbf{5 a})$.

CCDC Deposit No.

Empirical formula

Formula weight

Temperature

Wavelength

Crystal system

Space group

Unit cell dimensions

Volume

Z

Density (calculated)

Absorption coefficient

$\mathrm{F}(000)$

Crystal size

$\theta$ range for data collection

Index ranges

Reflections collected

Independent reflections

Completeness to $\theta=25.242^{\circ}$

Absorption correction

Max. and min. transmission

Refinement method

Data / restraints / parameters

Goodness-of-fit on $\mathrm{F}^{2}$

Final $\mathrm{R}$ indices $[\mathrm{I}>2 \sigma(\mathrm{I})]$

$\mathrm{R}$ indices (all data)

Largest diff. peak and hole
1453458

$\mathrm{C}_{20} \mathrm{H}_{24} \mathrm{O}_{8}$

392.39

150(2) K

$0.71073 \AA$

Monoclinic

$\mathrm{P} 2 / \mathrm{c}$

$\mathrm{a}=12.4356(6) \AA$

$\alpha=90^{\circ}$

$\mathrm{b}=20.9630(10) \AA$

$\mathrm{c}=7.8581(4) \AA$

1985.42(17) $\AA^{3}$

$\beta=104.2565(9)^{\circ}$

$\gamma=90^{\circ}$
4

$1.313 \mathrm{Mg} / \mathrm{m}^{3}$

$0.102 \mathrm{~mm}^{-1}$

832

$0.170 \times 0.160 \times 0.070 \mathrm{~mm}^{3}$

1.690 to $28.326^{\circ}$

$-16 \leq \mathrm{h} \leq 16,-27 \leq \mathrm{k} \leq 27,-10 \leq 1 \leq 10$

52534

$4960[\mathrm{R}(\mathrm{int})=0.0595]$

$100.0 \%$

Semi-empirical from equivalents

0.928 and 0.895

Full-matrix least-squares on $\mathrm{F}^{2}$

4960 / 0 / 257

1.033

$\mathrm{R} 1=0.0415, \mathrm{wR} 2=0.0950$

$\mathrm{R} 1=0.0638, \mathrm{wR} 2=0.1070$

0.448 and $-0.300 \mathrm{e}^{-3}$ 
Table S2. Atomic coordinates $\left[\times 10^{4}\right]$ and equivalent isotropic displacement parameters $\left[\AA^{2} \times 10^{3}\right]$ for $\mathrm{C}_{20} \mathrm{H}_{24} \mathrm{O}_{8}(\mathbf{5 a})$. $\mathrm{U}(\mathrm{eq})$ is defined as one third of the trace of the orthogonalized $\mathrm{U}_{\mathrm{ij}}$ tensor.

\begin{tabular}{|c|c|c|c|c|}
\hline & $\mathrm{x}$ & $\mathrm{y}$ & z & $\mathrm{U}(\mathrm{eq})$ \\
\hline $\mathrm{O}(1)$ & $3052(1)$ & $4354(1)$ & $1103(1)$ & $29(1)$ \\
\hline $\mathrm{O}(2)$ & $2247(1)$ & $3605(1)$ & 2411(1) & $24(1)$ \\
\hline $\mathrm{O}(3)$ & 654(1) & $3819(1)$ & $5928(1)$ & $31(1)$ \\
\hline $\mathrm{O}(4)$ & $542(1)$ & $4411(1)$ & $3502(1)$ & $27(1)$ \\
\hline $\mathrm{O}(5)$ & $5930(1)$ & $3925(1)$ & $8915(2)$ & $34(1)$ \\
\hline $\mathrm{O}(6)$ & $4487(1)$ & $3261(1)$ & $8669(1)$ & $26(1)$ \\
\hline $\mathrm{O}(7)$ & $2785(1)$ & $6675(1)$ & $6179(2)$ & $40(1)$ \\
\hline $\mathrm{O}(8)$ & 2177(1) & $6317(1)$ & $3425(2)$ & $38(1)$ \\
\hline $\mathrm{C}(1)$ & $2739(1)$ & $4567(1)$ & $3940(2)$ & $20(1)$ \\
\hline$C(2)$ & $2307(1)$ & $4276(1)$ & $5371(2)$ & $20(1)$ \\
\hline$C(3)$ & $2932(1)$ & $4142(1)$ & $6966(2)$ & $22(1)$ \\
\hline $\mathrm{C}(4)$ & $4109(1)$ & $4316(1)$ & $7611(2)$ & $22(1)$ \\
\hline$C(5)$ & $4477(1)$ & $4920(1)$ & $7683(2)$ & $25(1)$ \\
\hline$C(6)$ & $3826(1)$ & $5492(1)$ & $7071(2)$ & $26(1)$ \\
\hline$C(7)$ & $3216(1)$ & $5609(1)$ & $5451(2)$ & $23(1)$ \\
\hline $\mathrm{C}(8)$ & $3099(1)$ & $5166(1)$ & $3958(2)$ & $22(1)$ \\
\hline $\mathrm{C}(9)$ & $2703(1)$ & $4180(1)$ & $2330(2)$ & $20(1)$ \\
\hline$C(10)$ & $2142(1)$ & $3207(1)$ & $863(2)$ & $27(1)$ \\
\hline $\mathrm{C}(11)$ & $1796(2)$ & $2552(1)$ & $1313(2)$ & $34(1)$ \\
\hline$C(12)$ & 1091(1) & 4131(1) & $4992(2)$ & $22(1)$ \\
\hline$C(13)$ & $-654(1)$ & $4314(1)$ & $3008(2)$ & $33(1)$ \\
\hline$C(14)$ & $-1105(1)$ & 4693(1) & $1367(2)$ & $43(1)$ \\
\hline$C(15)$ & 4944(1) & $3826(1)$ & $8462(2)$ & $24(1)$ \\
\hline$C(16)$ & $5283(1)$ & $2774(1)$ & $9516(2)$ & $31(1)$ \\
\hline$C(17)$ & $4663(1)$ & $2159(1)$ & $9482(2)$ & $36(1)$ \\
\hline $\mathrm{C}(18)$ & $2714(1)$ & $6260(1)$ & $5097(2)$ & $28(1)$ \\
\hline C(19) & $1621(2)$ & $6923(1)$ & $2916(3)$ & $54(1)$ \\
\hline $\mathrm{C}(20)$ & $1035(2)$ & $6877(1)$ & $1066(4)$ & $81(1)$ \\
\hline $\mathrm{H}(3 \mathrm{~A})$ & 2593 & 3917 & 7746 & 26 \\
\hline $\mathrm{H}(5 \mathrm{~A})$ & 5247 & 4982 & 8187 & 30 \\
\hline $\mathrm{H}(6 \mathrm{~A})$ & 3838 & 5816 & 7920 & 31 \\
\hline $\mathrm{H}(8 \mathrm{~A})$ & 3294 & 5319 & 2937 & 26 \\
\hline $\mathrm{H}(10 \mathrm{~A})$ & 2859 & 3184 & 531 & 32 \\
\hline $\mathrm{H}(10 \mathrm{~B})$ & 1578 & 3386 & -137 & 32 \\
\hline $\mathrm{H}(11 \mathrm{~A})$ & 1687 & 2275 & 280 & 51 \\
\hline $\mathrm{H}(11 \mathrm{~B})$ & 1101 & 2583 & 1683 & 51 \\
\hline $\mathrm{H}(11 \mathrm{C})$ & 2375 & 2372 & 2271 & 51 \\
\hline $\mathrm{H}(13 \mathrm{~A})$ & -988 & 4461 & 3960 & 39 \\
\hline $\mathrm{H}(13 \mathrm{~B})$ & -827 & 3856 & 2791 & 39 \\
\hline $\mathrm{H}(14 \mathrm{~A})$ & -1909 & 4631 & 980 & 64 \\
\hline $\mathrm{H}(14 \mathrm{~B})$ & -756 & 4550 & 444 & 64 \\
\hline
\end{tabular}




$\begin{array}{lrrrr}\mathrm{H}(14 \mathrm{C}) & -945 & 5147 & 1606 & 64 \\ \mathrm{H}(16 \mathrm{~A}) & 5632 & 2899 & 10743 & 37 \\ \mathrm{H}(16 \mathrm{~B}) & 5873 & 2726 & 8880 & 37 \\ \mathrm{H}(17 \mathrm{~A}) & 5173 & 1827 & 10081 & 55 \\ \mathrm{H}(17 \mathrm{~B}) & 4346 & 2031 & 8262 & 55 \\ \mathrm{H}(17 \mathrm{C}) & 4065 & 2216 & 10081 & 55 \\ \mathrm{H}(19 \mathrm{~A}) & 2171 & 7273 & 3090 & 65 \\ \mathrm{H}(19 \mathrm{C}) & 1090 & 7011 & 3641 & 65 \\ \mathrm{H}(20 \mathrm{D}) & 635 & 7276 & 694 & 122 \\ \mathrm{H}(20 \mathrm{~A}) & 506 & 6522 & 905 & 122 \\ \mathrm{H}(20 \mathrm{~B}) & 1571 & 6802 & 358 & 122\end{array}$


Table S3. Bond lengths $[\AA]$ and angles $\left[^{\circ}\right]$ for $\mathrm{C}_{20} \mathrm{H}_{24} \mathrm{O}_{8}(\mathbf{5 a})$.

$\begin{array}{lcll}\mathrm{O}(1)-\mathrm{C}(9) & 1.2077(16) & \mathrm{O}(2)-\mathrm{C}(9) & 1.3399(16) \\ \mathrm{O}(2)-\mathrm{C}(10) & 1.4543(16) & \mathrm{O}(3)-\mathrm{C}(12) & 1.2080(17) \\ \mathrm{O}(4)-\mathrm{C}(12) & 1.3369(17) & \mathrm{O}(4)-\mathrm{C}(13) & 1.4552(17) \\ \mathrm{O}(5)-\mathrm{C}(15) & 1.2080(17) & \mathrm{O}(6)-\mathrm{C}(15) & 1.3412(17) \\ \mathrm{O}(6)-\mathrm{C}(16) & 1.4633(17) & \mathrm{O}(7)-\mathrm{C}(18) & 1.2054(18) \\ \mathrm{O}(8)-\mathrm{C}(18) & 1.3249(19) & \mathrm{O}(8)-\mathrm{C}(19) & 1.454(2) \\ \mathrm{C}(1)-\mathrm{C}(8) & 1.3326(19) & \mathrm{C}(1)-\mathrm{C}(2) & 1.4903(18) \\ \mathrm{C}(1)-\mathrm{C}(9) & 1.4948(19) & \mathrm{C}(2)-\mathrm{C}(3) & 1.3322(19) \\ \mathrm{C}(2)-\mathrm{C}(12) & 1.4992(19) & \mathrm{C}(3)-\mathrm{C}(4) & 1.4724(19) \\ \mathrm{C}(4)-\mathrm{C}(5) & 1.343(2) & \mathrm{C}(4)-\mathrm{C}(15) & 1.495(2) \\ \mathrm{C}(5)-\mathrm{C}(6) & 1.461(2) & \mathrm{C}(6)-\mathrm{C}(7) & 1.335(2) \\ \mathrm{C}(7)-\mathrm{C}(8) & 1.4744(19) & \mathrm{C}(7)-\mathrm{C}(18) & 1.498(2) \\ \mathrm{C}(10)-\mathrm{C}(11) & 1.507(2) & \mathrm{C}(13)-\mathrm{C}(14) & 1.501(2) \\ \mathrm{C}(16)-\mathrm{C}(17) & 1.499(2) & \mathrm{C}(19)-\mathrm{C}(20) & 1.460(3) \\ & & & \\ \mathrm{C}(9)-\mathrm{O}(2)-\mathrm{C}(10) & 115.35(10) & \mathrm{C}(12)-\mathrm{O}(4)-\mathrm{C}(13) & 116.15(11) \\ \mathrm{C}(15)-\mathrm{O}(6)-\mathrm{C}(16) & 114.32(11) & \mathrm{C}(18)-\mathrm{O}(8)-\mathrm{C}(19) & 116.20(13) \\ \mathrm{C}(8)-\mathrm{C}(1)-\mathrm{C}(2) & 124.07(12) & \mathrm{C}(8)-\mathrm{C}(1)-\mathrm{C}(9) & 117.46(12) \\ \mathrm{C}(2)-\mathrm{C}(1)-\mathrm{C}(9) & 118.35(11) & \mathrm{C}(3)-\mathrm{C}(2)-\mathrm{C}(1) & 124.13(12) \\ \mathrm{C}(3)-\mathrm{C}(2)-\mathrm{C}(12) & 118.03(12) & \mathrm{C}(1)-\mathrm{C}(2)-\mathrm{C}(12) & 117.83(11) \\ \mathrm{C}(2)-\mathrm{C}(3)-\mathrm{C}(4) & 124.88(13) & \mathrm{C}(5)-\mathrm{C}(4)-\mathrm{C}(3) & 123.21(13) \\ \mathrm{C}(5)-\mathrm{C}(4)-\mathrm{C}(15) & 115.88(13) & \mathrm{C}(3)-\mathrm{C}(4)-\mathrm{C}(15) & 120.40(12) \\ \mathrm{C}(4)-\mathrm{C}(5)-\mathrm{C}(6) & 127.20(13) & \mathrm{C}(7)-\mathrm{C}(6)-\mathrm{C}(5) & 127.15(13) \\ \mathrm{C}(6)-\mathrm{C}(7)-\mathrm{C}(8) & 124.36(13) & \mathrm{C}(6)-\mathrm{C}(7)-\mathrm{C}(18) & 117.23(13) \\ \mathrm{C}(8)-\mathrm{C}(7)-\mathrm{C}(18) & 118.10(13) & \mathrm{C}(1)-\mathrm{C}(8)-\mathrm{C}(7) & 124.69(12) \\ \mathrm{O}(1)-\mathrm{C}(9)-\mathrm{O}(2) & 123.38(12) & \mathrm{O}(1)-\mathrm{C}(9)-\mathrm{C}(1) & 124.71(12) \\ \mathrm{O}(2)-\mathrm{C}(9)-\mathrm{C}(1) & 111.91(11) & \mathrm{O}(2)-\mathrm{C}(10)-\mathrm{C}(11) & 107.34(11) \\ \mathrm{O}(3)-\mathrm{C}(12)-\mathrm{O}(4) & 124.16(13) & \mathrm{O}(3)-\mathrm{C}(12)-\mathrm{C}(2) & 124.87(13) \\ \mathrm{O}(4)-\mathrm{C}(12)-\mathrm{C}(2) & 110.93(11) & \mathrm{O}(4)-\mathrm{C}(13)-\mathrm{C}(14) & 107.31(13) \\ \mathrm{O}(5)-\mathrm{C}(15)-\mathrm{O}(6) & 123.05(13) & \mathrm{O}(5)-\mathrm{C}(15)-\mathrm{C}(4) & 123.87(13) \\ \mathrm{O}(6)-\mathrm{C}(15)-\mathrm{C}(4) & 113.08(12) & \mathrm{O}(6)-\mathrm{C}(16)-\mathrm{C}(17) & 107.75(12) \\ \mathrm{O}(7)-\mathrm{C}(18)-\mathrm{O}(8) & 124.43(14) & \mathrm{O}(7)-\mathrm{C}(18)-\mathrm{C}(7) & 124.96(15) \\ \mathrm{O}(8)-\mathrm{C}(18)-\mathrm{C}(7) & 110.61(12) & \mathrm{O}(8)-\mathrm{C}(19)-\mathrm{C}(20) & 107.64(17)\end{array}$


Table S4. Anisotropic displacement parameters $\left[\AA^{2} \times 10^{3}\right]$ for $\mathrm{C}_{20} \mathrm{H}_{24} \mathrm{O}_{8}$ (5a). The anisotropic displacement factor exponent takes the form: $-2 \pi^{2}\left[h{ }^{2} a^{* 2} U_{11}+\ldots+2 h k a * b * U_{12}\right]$

$\begin{array}{lrrrrrr} & \mathrm{U}_{11} & \mathrm{U}_{22} & \mathrm{U}_{33} & \mathrm{U}_{23} & \mathrm{U}_{13} & \mathrm{U}_{12} \\ \mathrm{O}(1) & 38(1) & 29(1) & 22(1) & -1(1) & 12(1) & -8(1) \\ \mathrm{O}(2) & 33(1) & 18(1) & 23(1) & -3(1) & 11(1) & -2(1) \\ \mathrm{O}(3) & 28(1) & 32(1) & 37(1) & 8(1) & 12(1) & -3(1) \\ \mathrm{O}(4) & 21(1) & 31(1) & 28(1) & 3(1) & 5(1) & 0(1) \\ \mathrm{O}(5) & 26(1) & 35(1) & 36(1) & 6(1) & 2(1) & -3(1) \\ \mathrm{O}(6) & 26(1) & 24(1) & 29(1) & 4(1) & 5(1) & 1(1) \\ \mathrm{O}(7) & 51(1) & 22(1) & 48(1) & -8(1) & 14(1) & 2(1) \\ \mathrm{O}(8) & 48(1) & 25(1) & 38(1) & 3(1) & 5(1) & 9(1) \\ \mathrm{C}(1) & 19(1) & 21(1) & 20(1) & 1(1) & 6(1) & 0(1) \\ \mathrm{C}(2) & 24(1) & 15(1) & 22(1) & -1(1) & 8(1) & 0(1) \\ \mathrm{C}(3) & 26(1) & 19(1) & 22(1) & 0(1) & 10(1) & -1(1) \\ \mathrm{C}(4) & 26(1) & 25(1) & 16(1) & 1(1) & 6(1) & -1(1) \\ \mathrm{C}(5) & 26(1) & 28(1) & 20(1) & -2(1) & 7(1) & -3(1) \\ \mathrm{C}(6) & 30(1) & 22(1) & 28(1) & -6(1) & 11(1) & -6(1) \\ \mathrm{C}(7) & 25(1) & 17(1) & 29(1) & -1(1) & 12(1) & -3(1) \\ \mathrm{C}(8) & 23(1) & 21(1) & 22(1) & 2(1) & 9(1) & 0(1) \\ \mathrm{C}(9) & 20(1) & 19(1) & 21(1) & 1(1) & 4(1) & 1(1) \\ \mathrm{C}(10) & 37(1) & 23(1) & 21(1) & -4(1) & 9(1) & -4(1) \\ \mathrm{C}(11) & 50(1) & 24(1) & 29(1) & -5(1) & 10(1) & -8(1) \\ \mathrm{C}(12) & 24(1) & 18(1) & 24(1) & -2(1) & 8(1) & 1(1) \\ \mathrm{C}(13) & 20(1) & 42(1) & 36(1) & 1(1) & 6(1) & 0(1) \\ \mathrm{C}(14) & 30(1) & 57(1) & 38(1) & 5(1) & 2(1) & 7(1) \\ \mathrm{C}(15) & 28(1) & 27(1) & 16(1) & 0(1) & 6(1) & -2(1) \\ \mathrm{C}(16) & 31(1) & 29(1) & 31(1) & 5(1) & 4(1) & 6(1) \\ \mathrm{C}(17) & 37(1) & 29(1) & 44(1) & 9(1) & 11(1) & 5(1) \\ \mathrm{C}(18) & 28(1) & 22(1) & 36(1) & -1(1) & 12(1) & -2(1) \\ \mathrm{C}(19) & 64(1) & 31(1) & 63(1) & 10(1) & 8(1) & 15(1) \\ \mathrm{C}(20) & 75(2) & 54(1) & 91(2) & 28(1) & -22(1) & 5(1)\end{array}$


Table S5. Torsion angles $\left[^{\circ}\right]$ for $\mathrm{C}_{20} \mathrm{H}_{24} \mathrm{O}_{8}($ (5a).

$\begin{array}{lclr}\mathrm{C}(8)-\mathrm{C}(1)-\mathrm{C}(2)-\mathrm{C}(3) & -69.34(19) & \mathrm{C}(9)-\mathrm{C}(1)-\mathrm{C}(2)-\mathrm{C}(3) & 114.76(15) \\ \mathrm{C}(8)-\mathrm{C}(1)-\mathrm{C}(2)-\mathrm{C}(12) & 110.07(15) & \mathrm{C}(9)-\mathrm{C}(1)-\mathrm{C}(2)-\mathrm{C}(12) & -65.83(16) \\ \mathrm{C}(1)-\mathrm{C}(2)-\mathrm{C}(3)-\mathrm{C}(4) & 6.3(2) & \mathrm{C}(12)-\mathrm{C}(2)-\mathrm{C}(3)-\mathrm{C}(4) & -173.15(12) \\ \mathrm{C}(2)-\mathrm{C}(3)-\mathrm{C}(4)-\mathrm{C}(5) & 58.5(2) & \mathrm{C}(2)-\mathrm{C}(3)-\mathrm{C}(4)-\mathrm{C}(15) & -130.11(15) \\ \mathrm{C}(3)-\mathrm{C}(4)-\mathrm{C}(5)-\mathrm{C}(6) & -3.7(2) & \mathrm{C}(15)-\mathrm{C}(4)-\mathrm{C}(5)-\mathrm{C}(6) & -175.45(13) \\ \mathrm{C}(4)-\mathrm{C}(5)-\mathrm{C}(6)-\mathrm{C}(7) & -57.7(2) & \mathrm{C}(5)-\mathrm{C}(6)-\mathrm{C}(7)-\mathrm{C}(8) & -0.3(2) \\ \mathrm{C}(5)-\mathrm{C}(6)-\mathrm{C}(7)-\mathrm{C}(18) & -173.82(14) & \mathrm{C}(2)-\mathrm{C}(1)-\mathrm{C}(8)-\mathrm{C}(7) & 5.7(2) \\ \mathrm{C}(9)-\mathrm{C}(1)-\mathrm{C}(8)-\mathrm{C}(7) & -178.37(12) & \mathrm{C}(6)-\mathrm{C}(7)-\mathrm{C}(8)-\mathrm{C}(1) & 56.6(2) \\ \mathrm{C}(18)-\mathrm{C}(7)-\mathrm{C}(8)-\mathrm{C}(1) & -129.97(15) & \mathrm{C}(10)-\mathrm{O}(2)-\mathrm{C}(9)-\mathrm{O}(1) & -3.20(19) \\ \mathrm{C}(10)-\mathrm{O}(2)-\mathrm{C}(9)-\mathrm{C}(1) & 177.74(11) & \mathrm{C}(8)-\mathrm{C}(1)-\mathrm{C}(9)-\mathrm{O}(1) & 5.8(2) \\ \mathrm{C}(2)-\mathrm{C}(1)-\mathrm{C}(9)-\mathrm{O}(1) & -178.00(13) & \mathrm{C}(8)-\mathrm{C}(1)-\mathrm{C}(9)-\mathrm{O}(2) & -175.12(12) \\ \mathrm{C}(2)-\mathrm{C}(1)-\mathrm{C}(9)-\mathrm{O}(2) & 1.05(17) & \mathrm{C}(9)-\mathrm{O}(2)-\mathrm{C}(10)-\mathrm{C}(11) & 170.87(12) \\ \mathrm{C}(13)-\mathrm{O}(4)-\mathrm{C}(12)-\mathrm{O}(3) & -1.0(2) & \mathrm{C}(13)-\mathrm{O}(4)-\mathrm{C}(12)-\mathrm{C}(2) & -178.95(12) \\ \mathrm{C}(3)-\mathrm{C}(2)-\mathrm{C}(12)-\mathrm{O}(3) & -11.2(2) & \mathrm{C}(1)-\mathrm{C}(2)-\mathrm{C}(12)-\mathrm{O}(3) & 169.38(13) \\ \mathrm{C}(3)-\mathrm{C}(2)-\mathrm{C}(12)-\mathrm{O}(4) & 166.74(12) & \mathrm{C}(1)-\mathrm{C}(2)-\mathrm{C}(12)-\mathrm{O}(4) & -12.71(17) \\ \mathrm{C}(12)-\mathrm{O}(4)-\mathrm{C}(13)-\mathrm{C}(14) & 177.12(13) & \mathrm{C}(16)-\mathrm{O}(6)-\mathrm{C}(15)-\mathrm{O}(5) & 0.88(19) \\ \mathrm{C}(16)-\mathrm{O}(6)-\mathrm{C}(15)-\mathrm{C}(4) & -179.37(11) & \mathrm{C}(5)-\mathrm{C}(4)-\mathrm{C}(15)-\mathrm{O}(5) & -13.1(2) \\ \mathrm{C}(3)-\mathrm{C}(4)-\mathrm{C}(15)-\mathrm{O}(5) & 174.89(13) & \mathrm{C}(5)-\mathrm{C}(4)-\mathrm{C}(15)-\mathrm{O}(6) & 167.17(12) \\ \mathrm{C}(3)-\mathrm{C}(4)-\mathrm{C}(15)-\mathrm{O}(6) & -4.85(18) & \mathrm{C}(15)-\mathrm{O}(6)-\mathrm{C}(16)-\mathrm{C}(17) & -174.27(12) \\ \mathrm{C}(19)-\mathrm{O}(8)-\mathrm{C}(18)-\mathrm{O}(7) & -1.5(2) & \mathrm{C}(19)-\mathrm{O}(8)-\mathrm{C}(18)-\mathrm{C}(7) & 178.24(14) \\ \mathrm{C}(6)-\mathrm{C}(7)-\mathrm{C}(18)-\mathrm{O}(7) & -3.1(2) & \mathrm{C}(8)-\mathrm{C}(7)-\mathrm{C}(18)-\mathrm{O}(7) & -177.03(14) \\ \mathrm{C}(6)-\mathrm{C}(7)-\mathrm{C}(18)-\mathrm{O}(8) & 177.16(13) & \mathrm{C}(8)-\mathrm{C}(7)-\mathrm{C}(18)-\mathrm{O}(8) & 3.21(18) \\ \mathrm{C}(18)-\mathrm{O}(8)-\mathrm{C}(19)-\mathrm{C}(20) & -177.03(18) & & \\ & & & \end{array}$

